\title{
DER UNTERGRUND DER KONTINENTE UND OZEANE NACH GEOPHYSIKALISCHEN UNTERSUCHUNGEN
}

\author{
Ludger Mintrop
}

Die geophysikalischen Untersuchungen über den Untergrund der Kontinente und Ozeane begannen mit Scbweremessungen. Sie zeigten zwei grossę Plänomene: Unter Gebirgen negative, auf den Meeren positive Bouguer-Anomalien. Erstere zeigen Massen-Defizite, letztere Massen-Überschüsse in der Erdkruste an. Im grossen und ganzen gesehen stellt der Verlauf der Kurve der Bouguer-Anomalien das Spiegelbild des Profiles der Oberfläche der festen Erdkruste dar, wie die unter Benutzung von Daten, die Heiskanen ( ${ }^{1}$ ) und Morelli ${ }^{(2}$ ) veröffentlicht haben, entworfenen Profile in den Figuren 1 und 2 zeigen.

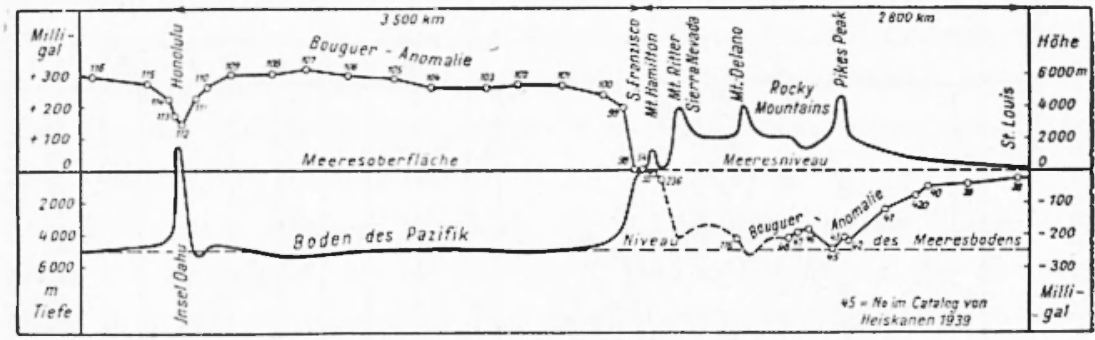

Fig. 1 - Bouguer-Schwere-Anomalien im Profil Rocky Mountains-Pazific.

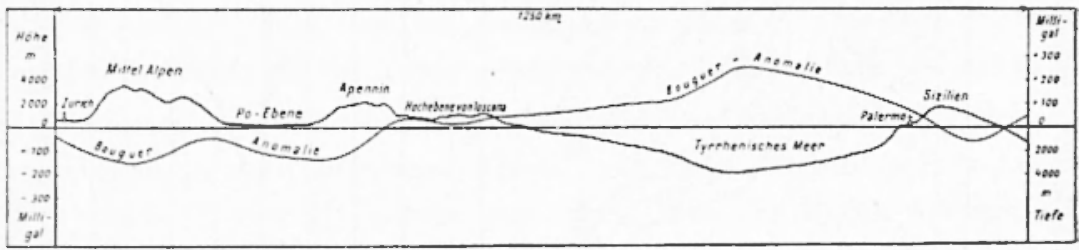

Fig. 2 - Bouguer-Schwere-Anomalien im Profil Alpen-Sizilien.

Wie die beiden Plänomene zu erklären sind, ist bis beute ein Mysterium geblieben, da der Vorgang des Lastenausgleiches in der Erde noch immer unbekannt ist. Zwar sind verschiedene Theorien dariiber auf gestellt worden; der experimentelle Nachweis des "Sitzes " des Massen-Defizits oder des Massen-Überscbusses ist aber bisher nock 
nach Th. Niethammer(1921)
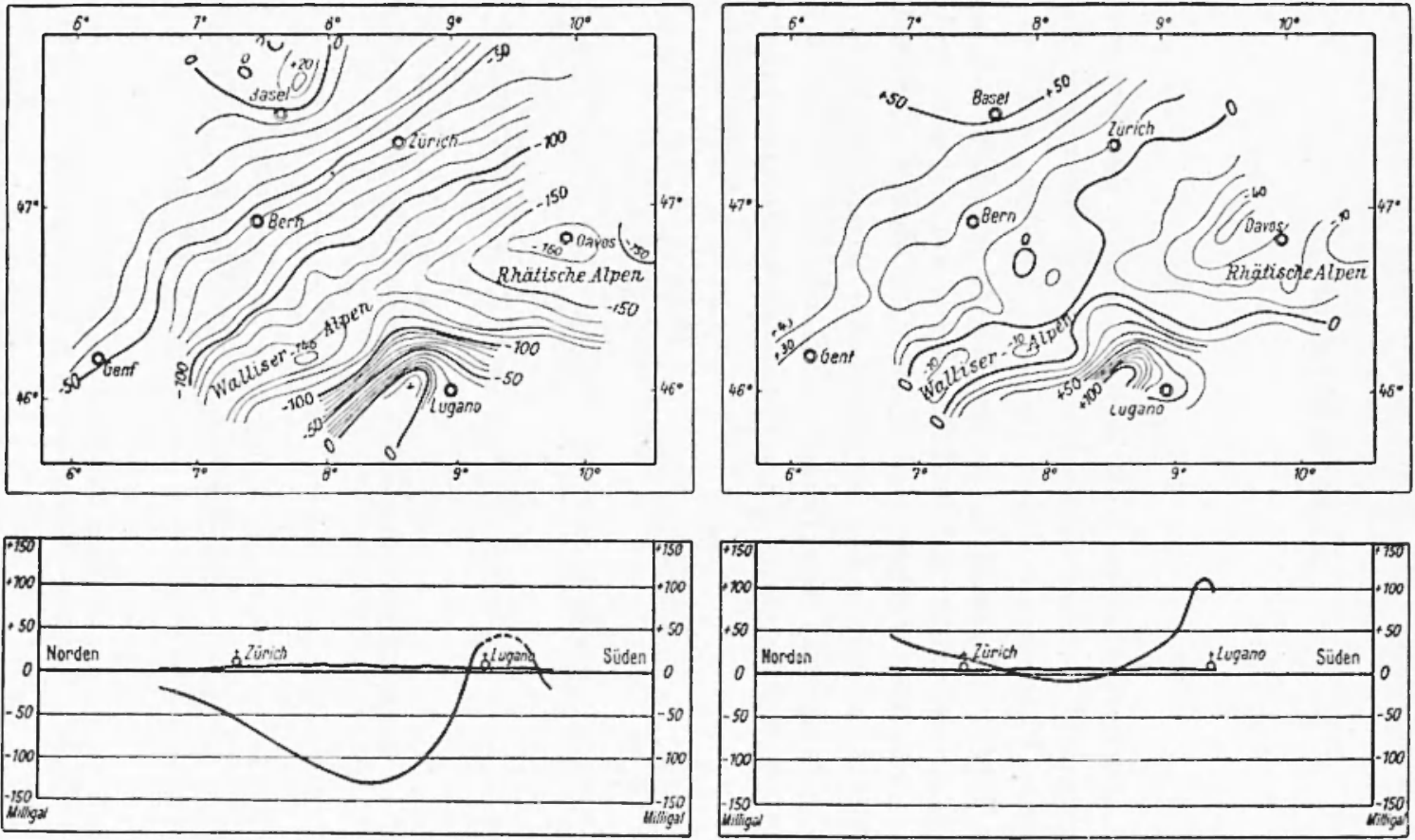

Profil der Bouguer-Schwere-Anomalien.

Profil der isostatischen Schwere-Anomalien.

fig. 3 - Bouguer-und isostatische Schwere-Anomalien in den Schweizer Alpen nach Niethammer. 
in keinem Falle gelungen. Zwei Hypothesen hahen seit fast loo Jabren im Vordergrund gestanden, die eine von Pratt $\left({ }^{3}\right)$, die andere von Airy $\left({ }^{4}\right)$. Ersterer nalım an, dass die Berge durch Expansion der Tiefenschichten nach oben getrieben worden seien und ihre Dichte entsprechend dieser Volumen-Vermehrung der Schichten geringer sei als ausserhalb des Gebirges. Nach Airy sollen die Berge in dichteren Substratum schwimmen, also von oben gekommen sein.

Für die Berechnung isostatischer Reduktionswerte in den Schweizer Alpen folgte Niethammer (") der Prattschen Vorstellung mit der Modifizierung von Hayford $\left({ }^{6}\right)$, wonach unter dem Gebirge bis zu einer (Ausgleichstiefe ) von $120 \mathrm{~km}$ eine gleicbmässige Dichtedifferenz angenommen wurde. In der Figur 3 sind neben den Bouguer-Anomalien die isostatischen Schwere-Anomalien wiedergegeben. Wie insbesondere die Profile in der Figur zeigen, sind durch die isostatische Reduktion zwar die grossen negativen Bouguer-Anomalien in der Achse des Gehirgszuges fast ganz verscbwunden, dafür treten aber nördlich der Alpen starke positive Anomalien auf, und an Südrande des Gehirges bereits vorhanden gewesene positive Anomalien werden erheblich verstärkt. Daraus ist ersichtlich, dass die den Berechnungen zu Grunde gelegte Vorstellung vom Massenausgleich der Wirklichkeit nicht entspricht. Das Dichte- Defizit ist offenbar mehr auf das Gebiet unter dem Kamm des Gebirges zu beschränken.

Mit einem solchen Ergebnis der Anwendung seiner Theorie hat Pratt ( ${ }^{7}$ ) gerechnet, als er schrieb: "The existence of the mountain mass is a fact indisputable. Not so the compensating cause, which is simply conjectural as to its existence, and altogether uncertain as to its extent, if it exist. We bave no certain and independed method of determining this, nor of ascertaining even if the hypothesis be valid how far down the attenuation extends or what law it follows".

Salonen $\left({ }^{s}\right)$ folgte bei seiner isostatischen Reduktion der Schweremessungen in den Schweizer Alpen nach dem Vorbild seines Lehrers Meistanen $\left({ }^{9}\right)$ der Hypothese von Airy. Wie die Figur 4 zeigt, wird die untere Grenze der unbelasteten Erde versuchsweise nacheinander zu 10 bis $30 \mathrm{~km}$ angenommen und der Querschnitt der "Wurzel" des Gebirges so geformt, dass die graphische Darstellung der Summe der Ergebnisse der Einzelrechnungen sich der Kurve der BouguerAnomalien möglichst anschliesst. Trotz aller Freiheiten, der Sulonen sich bei seinen Annahmen bedient, ist der Anschluss nur sehr unvollkonnnen gelungen. 
Profil II durch die Schweizer Alpen. (Abstand von den Profilen I u.lli je $25 \mathrm{~km}$ )
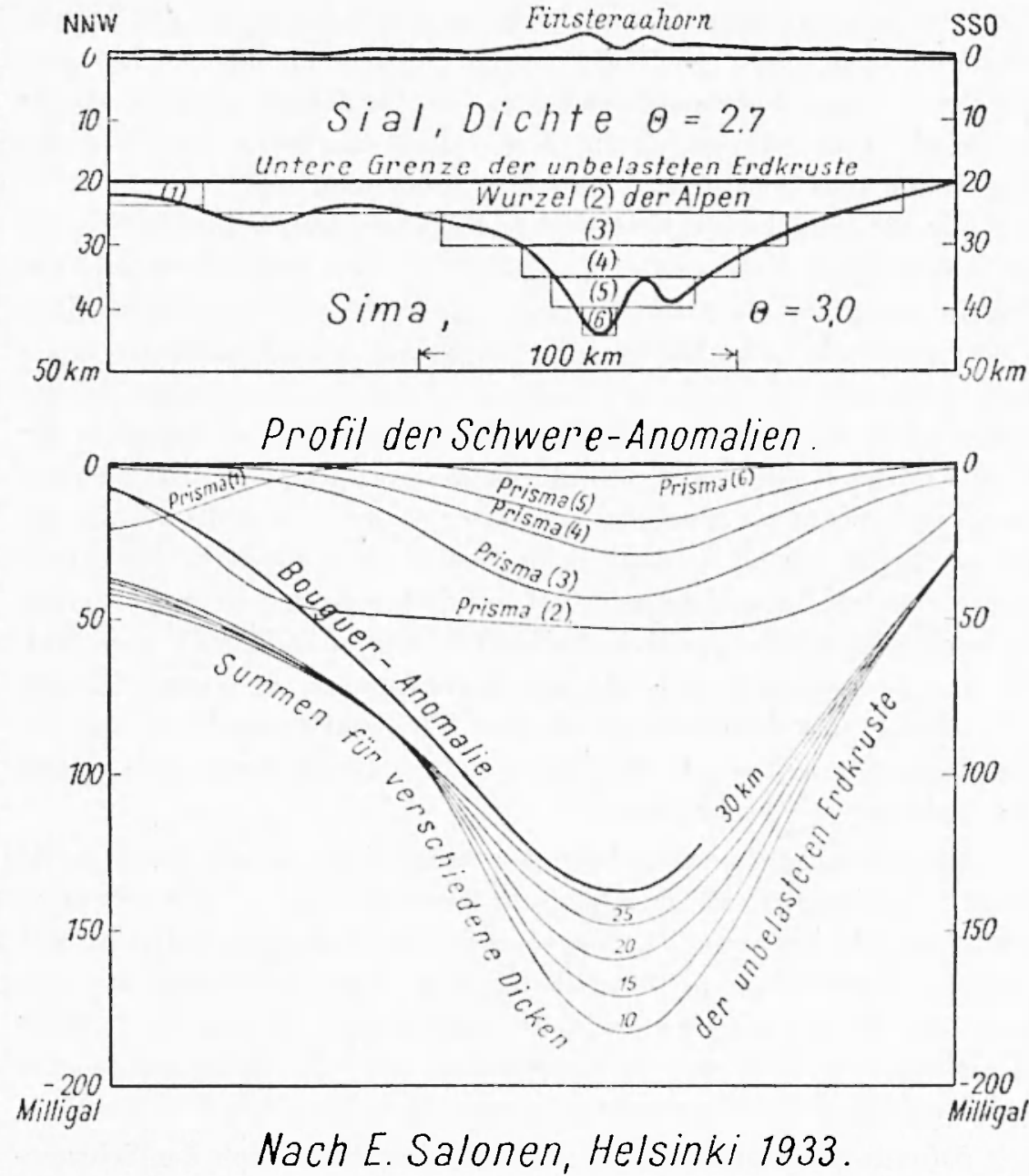

Fig. 4

Holopainen $\left({ }^{10}\right)$ hat versucht, die Wurzel der Zentral-Alpen zu ermitteln und ist dabei zu der im Profil der Figur 5 dargestellteu Form gekommen. Die Eintauchticfe ist dic yleiche wie bei Salonen, ohschon die Dichtedifferenz zwischen Sial und Sima doppelt so gross angenommen worden ist. Dieser Umstand allein hezeugt schon die Haltlosigkeit der Theorie von Airy. Letzterer hatte schon mit einer 


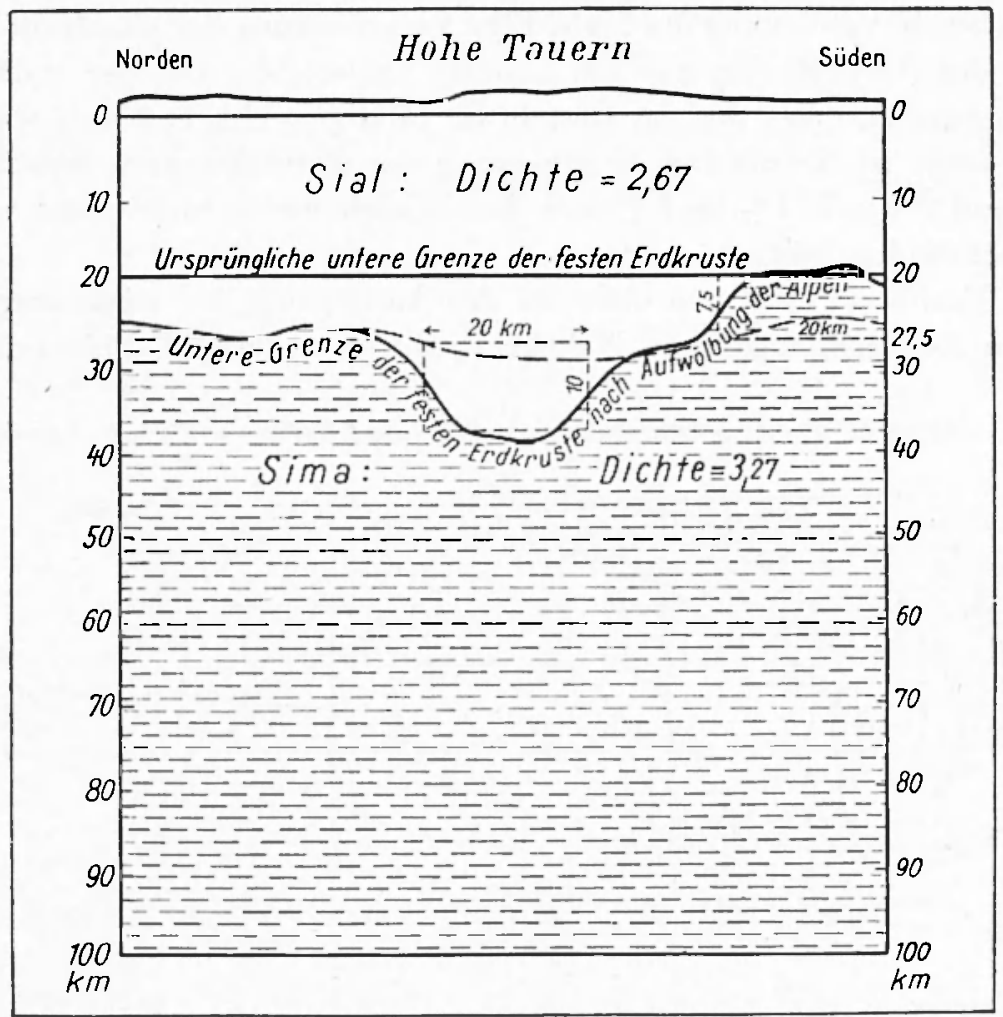

Fig. 5 - Protil durch die Zentral-Alpen nach Holopainen.

solchen Möglichkeit gerechnet, als er seine Ausführungen mit den Worten schloss: "In all the latter discussions it is supposed that the crust is floating in a state of equilibrium. But, in our entire ignorance of the modus operating of the forces which have raised sulmmarine strata to the tops of high mountains, we can not insist on this as absolutely true ".

Es liegen nun aber mehrere seismische Lintersuchungs-Ergebnisse vor, welche die "Wurzeltheorie " zu bestïtigen scheinen. Bei näherer Betrachtung erweisen sie sich aber entweder als nicht beweiskrätig oder als unrichtior.

Gutenberg ( $\left.{ }^{11}\right)$ schliesst in seiner Abhandhung "Seismological evidence for roots of mountains" aus einer Alonahme der normalen Zeitdifferenz zwischen den Ankunftszeiten der $P_{n}$ - und $\vec{P}$ - sowie zwischen der $P^{*}$ - und $P$ - Welle un etwa 2 Sckunden im Alpengebiet auf eine etwa $20 \mathrm{~km}$ dicke Wurzel des Gebirges, d. h. auf eine ent- 
sprechende Verdickung des Sials. Eine Vergrösserung der Gcschwindigkeit der $\bar{P}$-Welle um uur ein Zelıntel $\mathrm{km} / \mathrm{sec}$, die bei der starken Kompression, unter der das Gestein im Gehirge steht, mehr als wahrscheinlich ist, würde die Verringerung der Zeitdifferenzen zwischen $P_{\mathrm{n}}$ und $\bar{P}$ sowie $P^{*}$. und $\bar{P}$ und damit auch die Gehirgswurzel zum Verschwinden bringen.

Wanner $\left({ }^{12}\right)$ bat nun aber hei der Auswertung der seismograpbischen Aufzeichnungen von Nahbehcn, von denen das Walliser-Behen

Kurven der gleichen Schirereabweiclunng in Milligal nach Th. Niethammer K Schüfte u C Morelli.

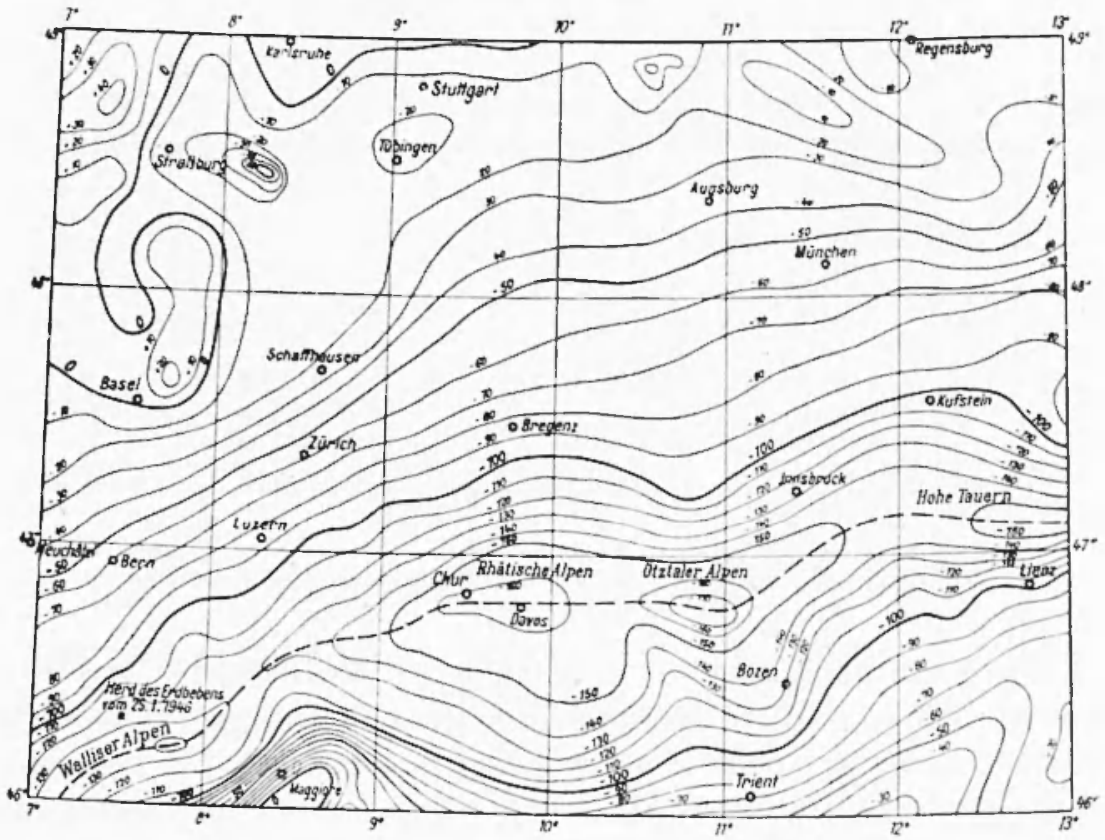

Fig. 6 - Linien gleicher Bouguer-Schwere-Anomalien in den Alpen und Lage des Epizentrums des Walliser Erdbebens vom 25. Januar 1946.

vom 25. Januar 1946 das stärkste war, ebenfalls Verzögerungen der Einsätze der $P_{n}$-Welle ermittelt und sie auf eine Gehirgswurzel zurückgefübrt. Die "Verzögerungen " betrugen in Basel $0.6 \mathrm{sec}$, in Zürich 1.4 sec und in Chur 2.1 see, waren also unter dem Gebirgskamm an grössten und fielen dort mit der grössten Bouguer-Anomalie zusammen (Fig. 6). Andererseits ergab sich in Neuchâtel ein Zeitgewinn von 1.1 sec, so dass der erste Einsatz nach der Ansicht von $W$ anner " sieher nicht die $P_{\mathrm{n}}-$ Welle sei ". Die Verzögerung in Basel wird als 


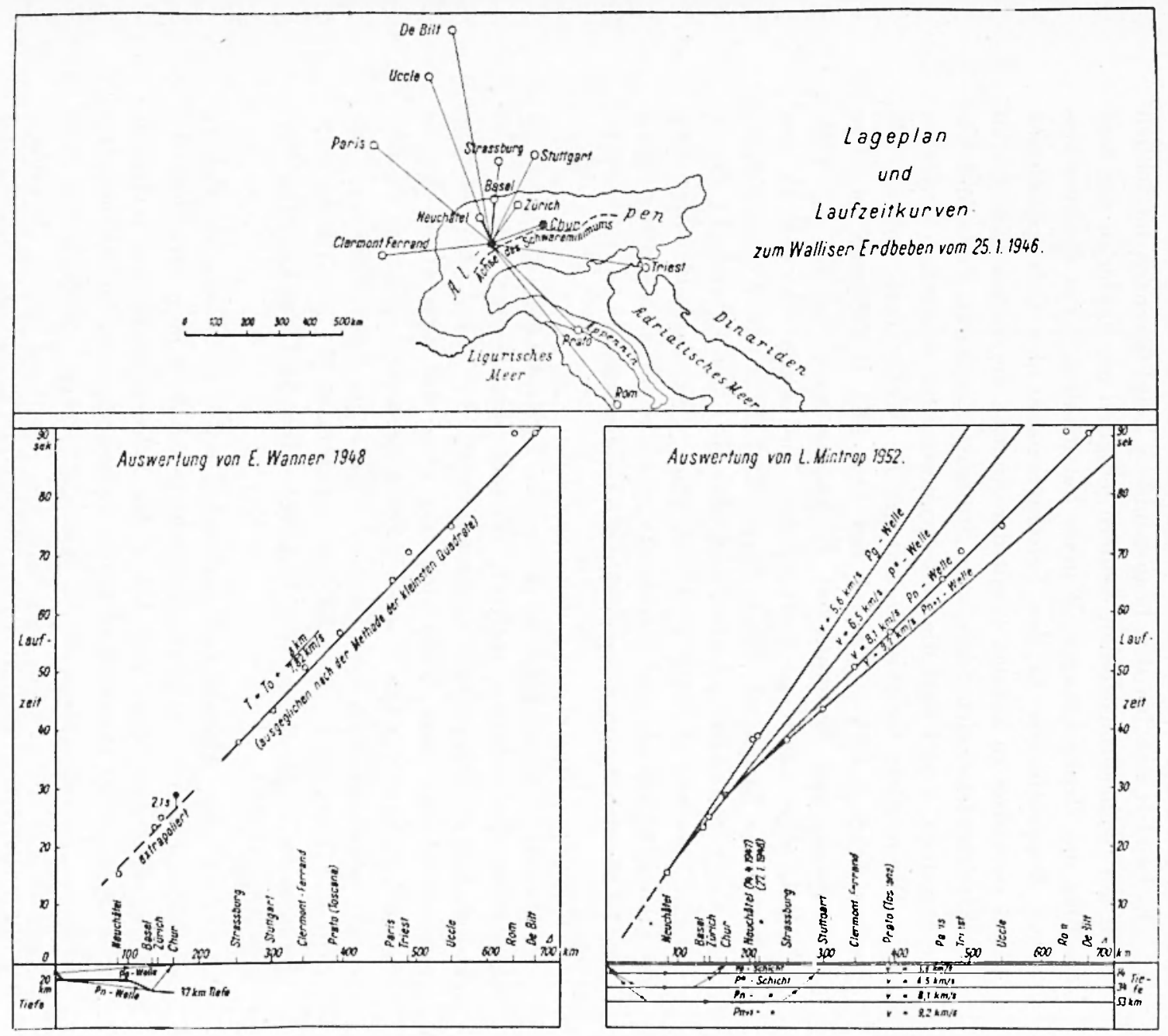

Fig. 7 - Lageplan und Laufzeitkurven zum Walliser Erdbeben vom 25.1.1946. 
in Bereich der unvermeidlichen Zeitfehler angesehen, diejenige in Zürich schon nicht mehr, während über die 2.1 sec in Chur als Beobachtungsfehler gar nicht diskutiert werden könnte.

Es handelt sich bei den heanstandeten Zeitdifferenzen tatsächlich nicht um Beohachtungsfehler, sondern lediglich um Fehler in der Auswertung der Beohachtungen. Tanner hat nämlich, " um die verschisdenen Doppeleinsäze in den Seismogrammen der Gebirogstationen hesser entwirren zu können ", mit der Analyse der ersten Einsâtze an den Flachlandstationen Strasslıurg, Stuttgart, Clermont, Paris und Uccle hegonnen, "weil dort die Untergrundstruktur wesentlich einfacher sei ». Die mittlere Geschwindigkeit der $P_{\mathrm{u}}$ - Welle ergah sich zu 7.82 $\mathrm{km} / \mathrm{sec}$ (Fig. 7 links). Tanner hat nun alle Differenzen zwischen Beobachtung und Rechnung auf die Laufzeitkurve für die $P_{n}-$ Welle hezogen, olne daran zu denken, dass die ersten Einsätze an den Nahstationen Neuchâtel, Basel, Zürich und Chur von Wellen herrühren, die in Schichten gelaufen sind, die über der $P_{n}$-Schicht liegen. (Figur 7 rechts). Manner geht von einer "Normaldicke " der Erdkruste von $20 \mathrm{~km}$ aus und berechnet den "Tiefgang der Alpenfaltun:r " zu $37 \mathrm{~km}$, wovon also $17 \mathrm{~km}$ auf die “Gehiregswurzel ) entfallen (Fig. 7 links).

Da die Verbindungslinie zwischen Epizentrum und Chur im Streichen des Gehirges verläuft, $T$ anner den Begim der Wurzel zwischen Epizentrum und Chur annimmt, die Achse des Schwereminimums aber nach Südwesten hin üher das Epizentrum hinaus verlauft, so kann die von $T$ anner herechnete Wurzel nicht die Ursache des Schwereminimums sein. Die von Tranner im Jahre 1930 veroffentlichten Laufzeiten zu dem $40 \mathrm{~km}$ östlich rom Walliser-Beben stattgefundenen Visper-Belsen vom 15.4.1924 zeigen in Chur keinerlei Verzögerung (Fig. 8).

Wenn eine Alpenwurzel vorhanden wäre, so müsste sie sich in den Laufzeitkurven bemerklsar machen, die quer zum Streichen des Gebirgzuges hzw. quer zur Achse des Schwereminimums verlaufen. Der Lageplan der Figur 9 enthält die Epizentren von fünf italienischen Erdbeben, deren Aufzeichnugen auf zahlreiclsen Stationen, darunter Rom, Pavia, Chur Zïrich und Stuttgart, von Caloi $\left({ }^{13}{ }^{14}\right)$, Di Filippo \& Marcelli ( $\left.{ }^{15,}{ }^{16}\right)$, Rosini ( ${ }^{17}$ ) und Valle ${ }^{18}$ ) einer sehr eingehenden Bearbeitung unterzogen worden sind. Der Verfasser hat die ron den genannten Autoren veröffentlichten Laufzeiten der $P_{\mathrm{g}}(=P)$-, $P^{*}$, und $P_{\mathrm{n}}$-Wellen in einem sehr grossen Masstabe in Ahhängigkeit ron der 


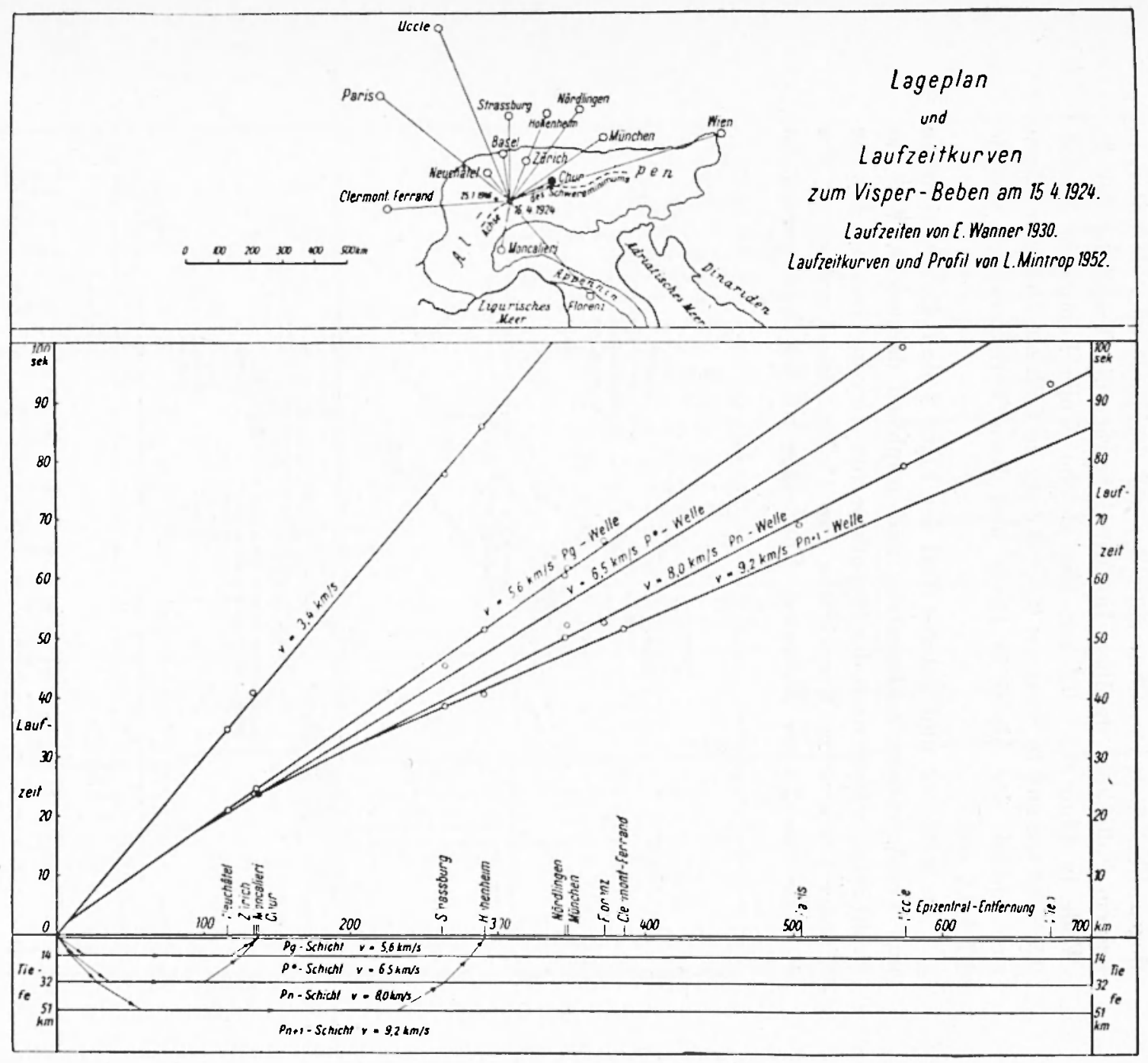

Fig. 8 - Lageplan und Laufzeitkurven zum Visper-Beben vom 15.4.1924. 
Epizentralentfernung dargestellt ( $1 \mathrm{sec}=4 \mathrm{~mm}, 1 \mathrm{~km}=1 \mathrm{~mm}$ ) und die Laufzeitkurven gezogen. In diesen geradlinig verlaufenden Kurven liegen die Zeiten von Chur entweder auf der Linie oder darunter, in keinem Falle aber darüber. Im Mittel beträgt die Voreilung der $P_{n}$ - Welle in Chur 0,3-0,2 sec. Die gleiche Welle kommt in Zürich $0,5+0,3$ sec und in Stuttgart $0,4-0,2$ sec zu früh an. Dagegen zeigen sich südlich der Alpen in Pavia und Rom Verzögerungen von je $0,7 \div 0,4$ sec.

Im mittleren und unteren Teil der Figur 9 sind die Voreilungen und Verzögerungen zahlenmässig und graphisch dargestellt. Wie das Profil zeigt, müsste nach der Hypothese von Airy in Chur bei der von Wanner berechneten Wurzeldicke von $17 \mathrm{~km}$ eine Verzögerung der $P_{\mathrm{o}}$ Welle von $2,3 \mathrm{sec}$ auftreten. Von einer solchen Verzögerung ist

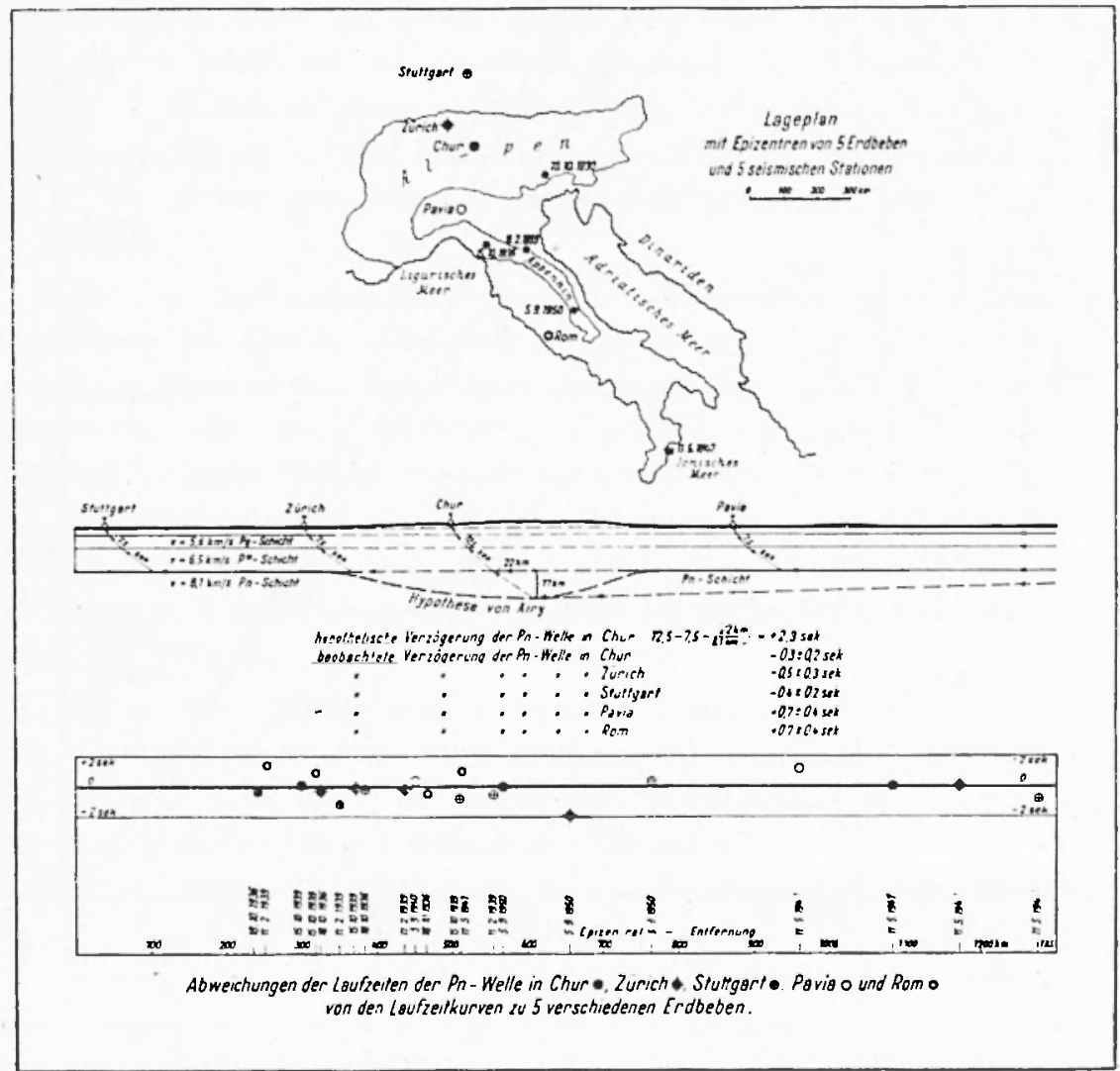

Fig. 9 - Lageplan mit Epizentren von 5 Erdbeben und 5 seismischen Stationen sowie Abweichungen der Laufzeiten der $P_{n}$. Welle. 

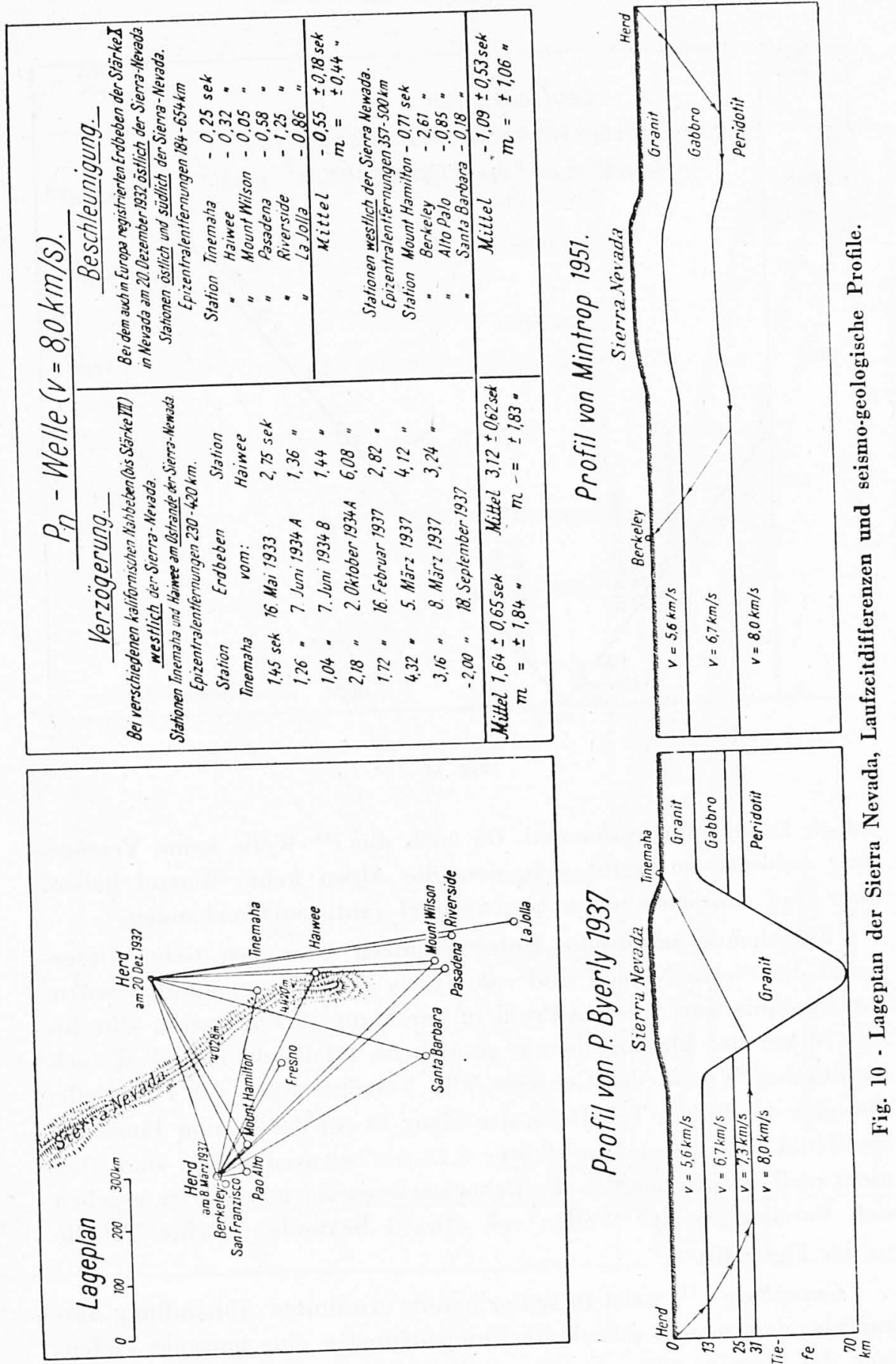


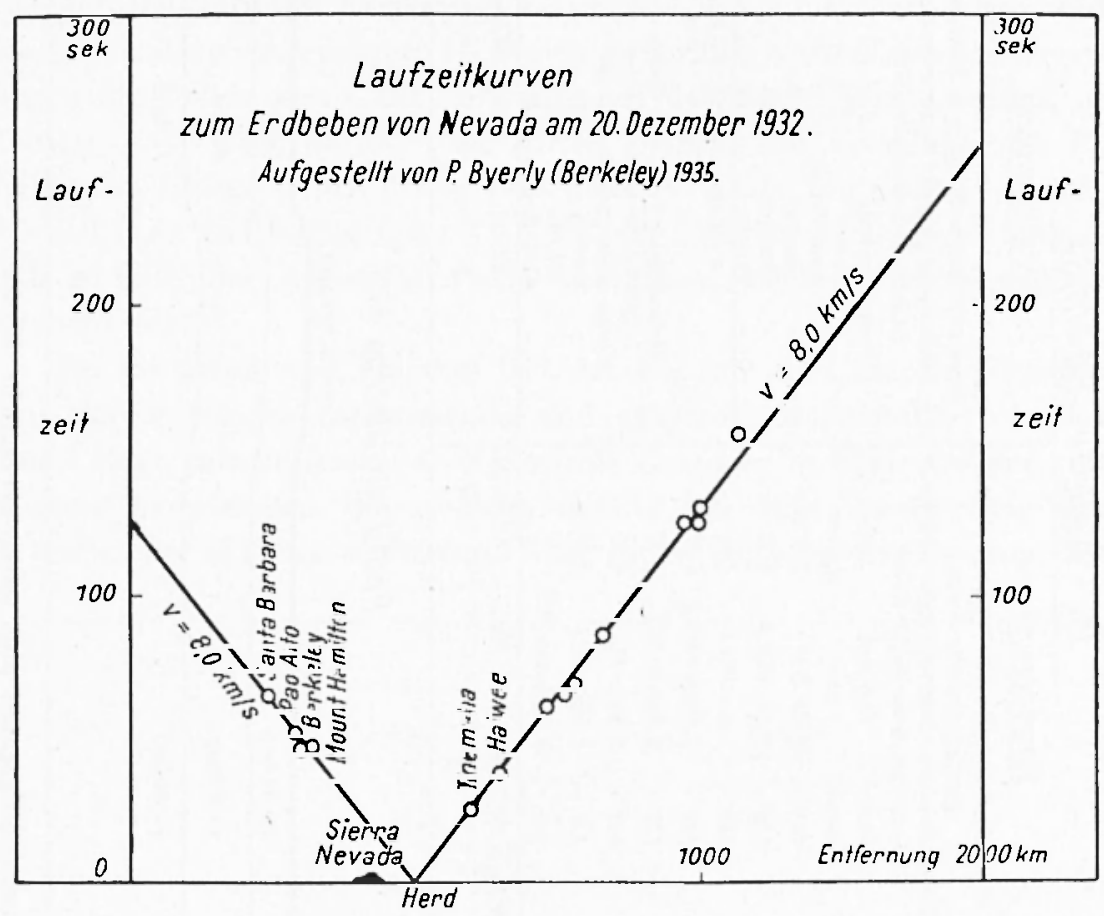

Fig. 11

jedoch keine Spur vorhanden. Da auch die $P^{*}$. Welle keine Verzögerung aufweist, so ergibt sich, dass die Alpen keine Wurzel hahen. Eher sind Anzeichen einer Gegenwurzel (anti-root) vorhanden.

Eingehende seismische Untersuchungen über den tiefen Untergrund der Sierra Nevada sind von Byerly (19, 20, 21) angestellt worden. Ibr Ergebnis war, wie das Profil in der Figur 10 links zeigt, eine bis fast $70 \mathrm{~km}$ tief hinabreichende granitische (vielleicht zum Teil auch basaltische) Wurzel des Gebirges. Die Verzogerungen der $P_{n}$-Welle, die nach der linken Tabelle in der Figur 10 auf der Station Tinemaha im Mittel 1.64 sec und in Haiwee 3.12 sec betragen sollen, sind aber nicht reell. Sobald nämlich die Bebcnstärke genügend gross ist, ergeben sich Voreilungen der Wellen von etwa 1 Sekunde (Rechte Tabelle in der Figur 10).

Gutenberg ( $\left.{ }^{11}\right)$ weist in seiner bereits erwähnten Abhandlung darauf hin, dass neuere seismische Untersuchungen eine normale Tiefenlage des Granits unter der Sierra Nevada ergeben hätten, aber An- 


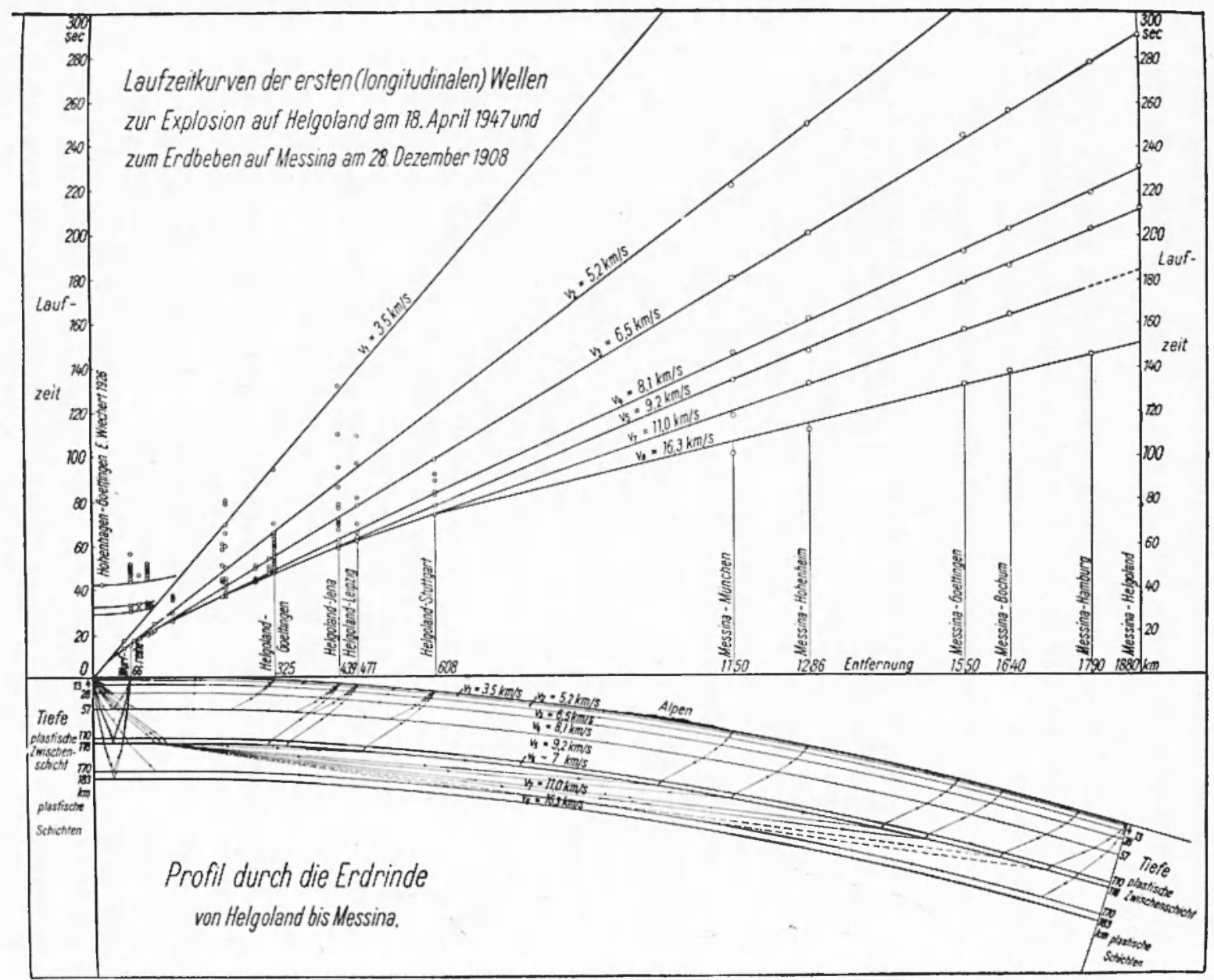

Fig. 12 - Ialufzeitkurven und seismo-geologisches Profil durch die Erdkruste nach Mintrop. 
zeichen für eine Verdickung der zwischen Granit und Peridotit (Wellengeschwindigkeit $8 \mathrm{~km} / \mathrm{sec}$ ) liegenden Schichten vorhanden seien. Die Laufzeitkurven für das Erdbeben von Nevada am 20. Dezember 1932, das die Stürke $X$ hatte, sind aber auf beiden Seiten, östlich und westlich, der Sierra Nevada (Fig. 11) gleich, so dass auch die " Basaltwirzel ) nicht vorhanden ist.

Die bisherigen seismischen Untersuchungen haben demnach keine Stiitze für die Richtigkeit der Airy'schen Annahme schwimmender Gebirge geliefert.

Seismisch ermitteltes Profil durch die Erdkruste.

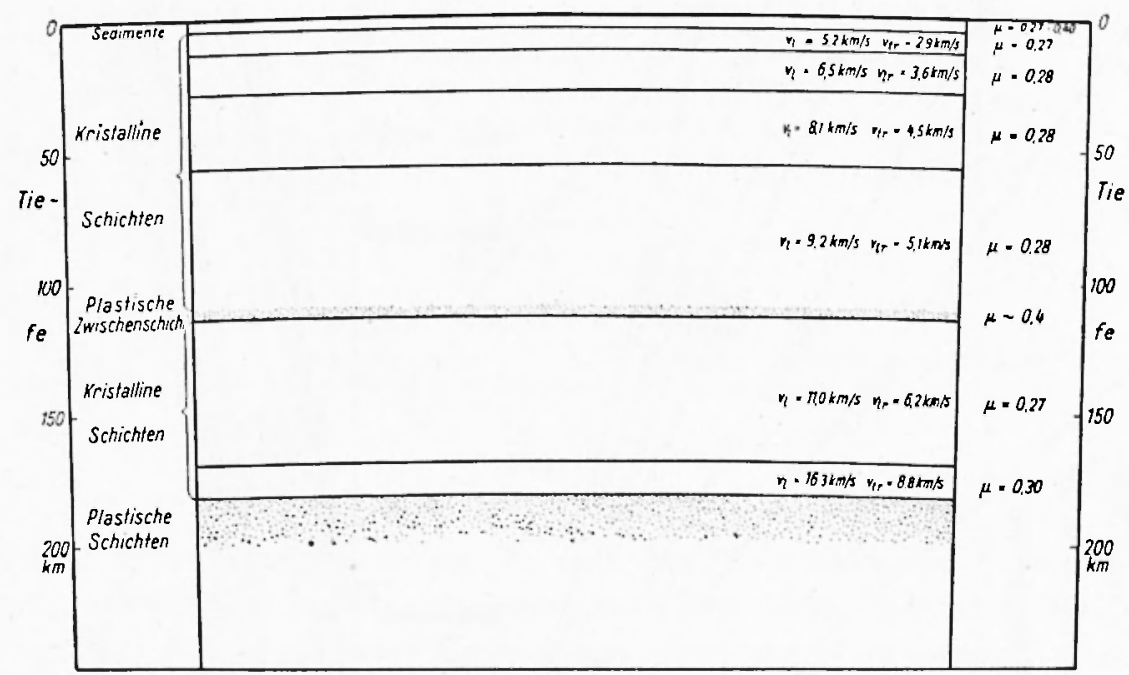

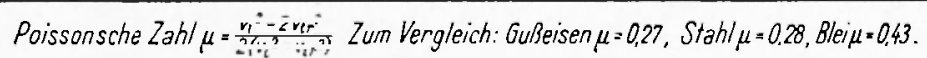

Fig. 13 - Seismo-geologisches Profil durch die Erdkruste nach Mintrop.

Eine grosse Wende in der seismographischen Erforschung der Erdkruste ist am 18. April 1947 eingetreten, an welchem Tage auf Helgoland vier Millionen Kilogramm $20 \mathrm{~m}$ unter der Oberfläche der Felseninsel in Tunneln lagernde Munition "in einem Schuss" zur Detonation gebracht worden sind. Dieses "künstliche Erdbeben " ist von vielen Seismologen sehr eingehend untersucht worden. Seine Aufzeichnungen in Deutschland und die Registrierungen des katastrophalen Erdbebens von Messina an 28. Dezember 1908 dienten dem Verfasser $\left({ }^{22}, 23\right)$ zur Aufstellung der in den Figuren 12 und 13 wiedergegebenen Profile durch die Erdkruste. Nach ibnen tritt die erste plastische 
Schicht erst in einer Tiefe von $110 \mathrm{~km}$ auf. Es ist deshall, ganz unmöglich, dass die Gehirge wie Eisherge in Suhstratum schwimmen. Das Sial kann gar nicht in das Sima eintauchen, weil letzteres kristallin und nicht etwa plastiscl, ist. Die Poisson'schen Zahlen sind, wie die Figur 13 zeigt, hei allen Sclichten (mit Ausnahme der plastischen) his zur Tiefe ron $170 \mathrm{~km}$ die gleichen wie für Stahl bei Zimmertemperatur. Die Wellengeschwindigkeiten für die plastische Zwischenschicht konnten nicht hestimmt werden, die Poisson'sche Zahl wird auf 0.4, etwa gleich der des Bleies, geschätzt.

Die für den Peridotit und für die darülser liegenden Schichten (Gahhro, Granit und Sedimente) vom Verfasser ermittelten Wellengeschwindigkeiten decken sich mit den Ergehnissen anderer Auswerter der Helgoland-Sprengung, die sich aher sämtlich auf die Untersuchung dieser Schichten lueschränkt hahen.

Uber die Schicht in $57 \mathrm{~km}$ Tiefe mit der Wellengeschwindigkeit $9.2 \mathrm{~km} / \mathrm{sec}$. hemerkte Angenheister ( $\left.{ }^{2+}\right)$ bereits im Jahre 1926 in einer Discussion auf dem Internationalen Geologen-Kongress in Madrid:

"Die seismischen Wellen erfahren eine selır gut ausgeprägte Geschwindigkeitszunahme in $60 \mathrm{~km}$ Tiefe. Dort muss eine elastische Discontinuität liegen. Zur Zeit werden in Deutschland Versuche gemacht, diese Discontinuitat experimentell durch Sprengungen festzustellen.

Stoneley ${ }^{(25)}$ schrieh in seiner Veroffentliehung iiher die Bearheitung der Laufzeiten zum Erdlselsen auf der griechischen Insel Kephallenia vom 18. Augrust 1926 üher die gleiche Schicht:

"Not all the large residuals, however, can he thus explained. Four points, fitting closely to a straight-line-graph (within $1.2 \mathrm{sec}$ as suhsequent computations prohes indicate a wawe travelling with the velocity $9.1 \mathrm{~km} / \mathrm{sec}$. These could be scarcely he the early $P$-wave of Mohorovicic, and the agrecment is doultful accidental. Presumahly microseism makes the heginning difficult to read ".

Mit einer plastischen Zwischenschicht in etwa loo $\mathrm{km}$ Tiefe ist von geoplysikalischer und geologischer Seite schon seit langer Zeit gerechnet worden. Tiechert ${ }^{(26)}$ hielt bereits 1897 für die Erklïrung der sichtharen Erhehungen und Einsenkungen der Erdoherfläche sowie der noch heute auf ihr stattindenden Massenverlagerungen eine plastische Zwischenschicht für erforderlich, die nur von verhaltnismässig geringer Dicke sein könnte. Hayford (6) nalım die Dicke zu $16 \mathrm{~km}$ an, Daly $\left({ }^{2 \pi}, 28\right)$ schatzt sie auf etwa $20 \mathrm{~km}$ hei einer Tiefcnlage ron 
rd $100 \mathrm{~km}$. Mohorovicic $\left({ }^{29}\right)$ hielt leereits im Jahre 1916 auf Grund von seismischen Messungen eine Discontinuitatsfläche in einer Tiefe von $120 \mathrm{~km}$ für höchstwahrscheinlich. Doelter $\left({ }^{30}\right)$ verlangte schon linnge levor Hayford ( $\left.{ }^{6}\right)$ und Helmert ( ${ }^{31}$ ) aus Lotablenkungen und Schweremessungen in 113 bzw. $118 \mathrm{~km}$ Tiefe eine isostatische Ausgleichsfläche angegeloen hatten und Mohorovicic seismische Anzeichen fand, eine Tiefe der primüren Vulkanberde von weniøstens $120 \mathrm{~km}$ Tiefe. Born ( $\left.{ }^{32}\right)$ nalım eine tatsächliche Ausgleichszone als Übergangrszone an der Unterseite der festen Erdkruste an, die viele Kilometer Mächtigkeit babe und in den einzelnen Krustenteilen verscbiedenl dick sei.

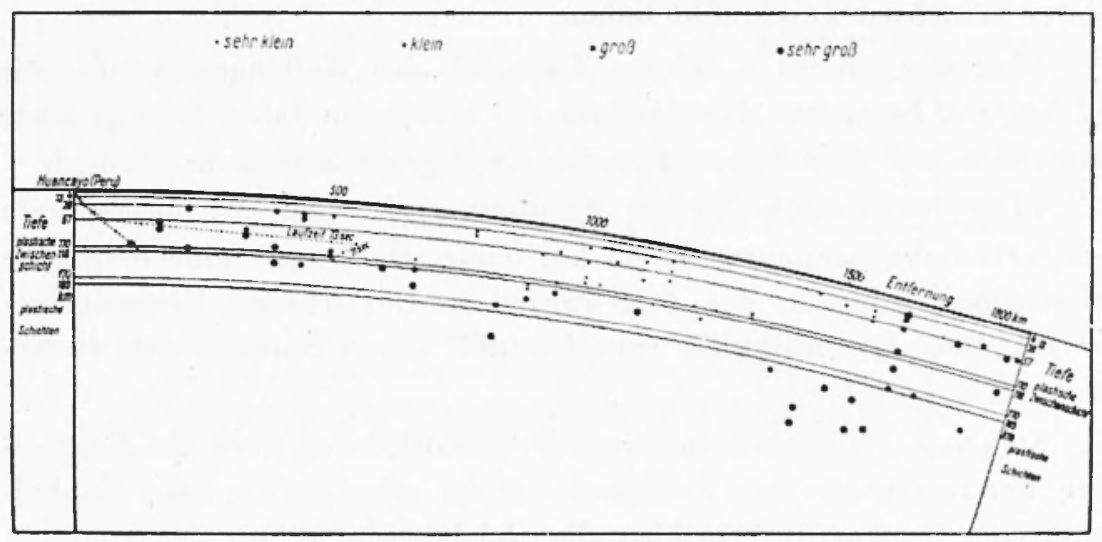

Fig. 14 - Seismo-geologisches Profil durch die Erdkruste nach Minirop und Amplituden von Vorlaufern zu Erdbeben in verschiedener Tiefe nach Gutenberg.

Gutenberg $\left({ }^{33}\right)$ und Richter haben in den in Huancayo registrierten Seismogrammen zahlreicher Erdbeben verschiedener EpizentralEntfernungen und unterschiedlicher Herdtiefen die Amplituden des ersten Einsatzes untersucht und sind dabei zu der Vermutung gekommen, dass sich in etwa $100 \mathrm{~km}$ Tiefe eine plastische Schicht befinden müsse, die sich in den Seismogrammen bzw. Laufzeitkurven als Schattenzone verrate. Wie das Profil der Figur 14 zeigt, fällt diese Zone mit der vom Verfasser gefundenen plastischen Schicht zusammen. Noch im Jahre 1938 hatte Daly $\left({ }^{27}\right)$ die Bestätigung dieser plastischen Schicht als eine Zukunftsaufgabe der Seismologen lezeichnet. 
In den Figuren 15 und 16 sind Seismogramme zum Erdheben von Messina reproduziert, in denen vor dem ersten scharfen Einsatz sehr, sehr kleine Vorwellen auftreten, deren Geschwindigkeit $11.0 \mathrm{~km} / \mathrm{sec}$ heträgt. Benndorf $\left(^{34}\right)$ heohachtete die gleiche Geschwindigkeit hereits im Jahre 1905 und Angenheister $\left({ }^{35}\right.$ j 1921. In dem von ihm mithe-

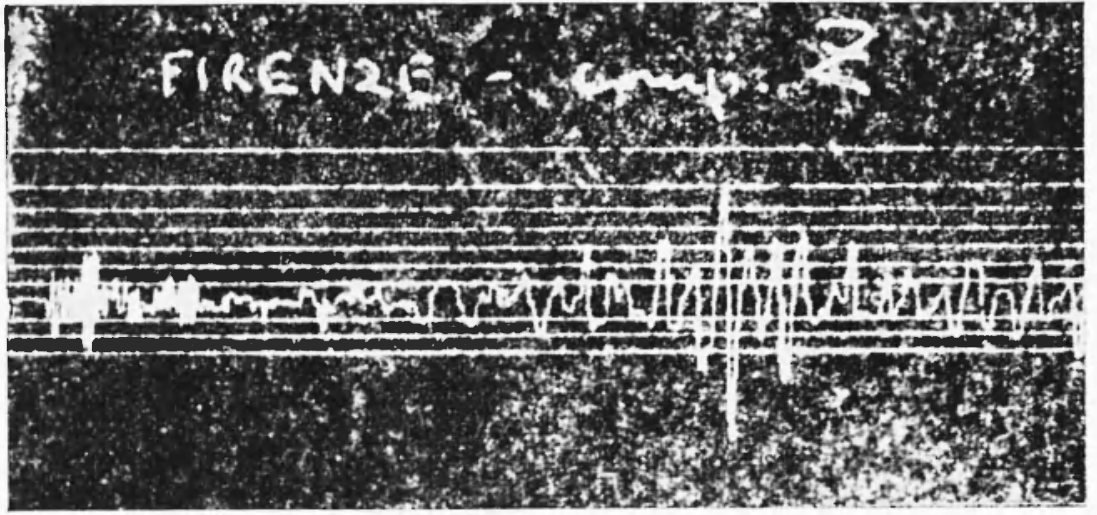

Fir. 15 - Vertical-Seismogramm von Florenz zum Erdbeben von Messina am 28. Dezember 1908.

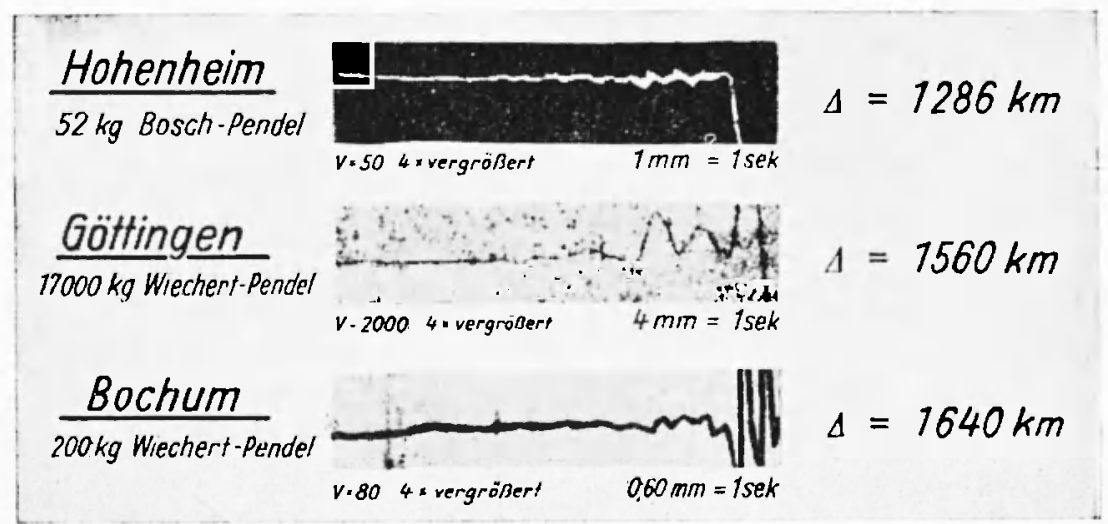

Fig. 16 - Vorläufer in Seismogrammen von Bochum, Göttingen und Hohenheim zum Erdbeben von Messina am 28. Dezember 1908.

nutzten in Apia aufgenommenen Seismogramm der Figur 17 zu einem Erdheben auf dem Tongaplateau fellt der schwache Voreinsatz, was darauf hindeuten konnte, dass die plastische Zwischenschicht, wenigtesns in diesem Teile des Pazific, fehlt.

Unter der $11.0 \mathrm{~km} / \mathrm{sec}$-Schicht liegt noch eine verhältnismässig dünne Schicht mit der enorm hohen Geschwindigkeit der longitudina- 
Thatimi Aria ( Jauna),

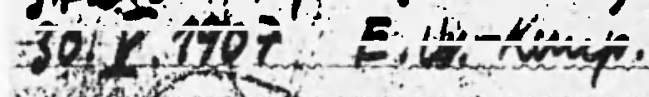

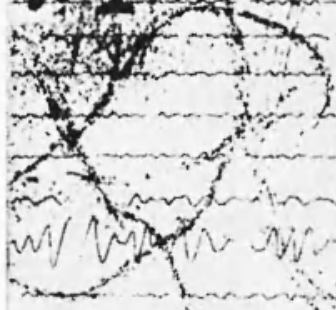

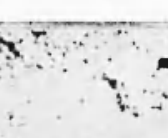

$\ddot{g}$

Herd: $-\phi=20,0^{\circ} S \lambda=176,8^{\circ} W$ (longarinne) $\triangle=882 \mathrm{~km}$

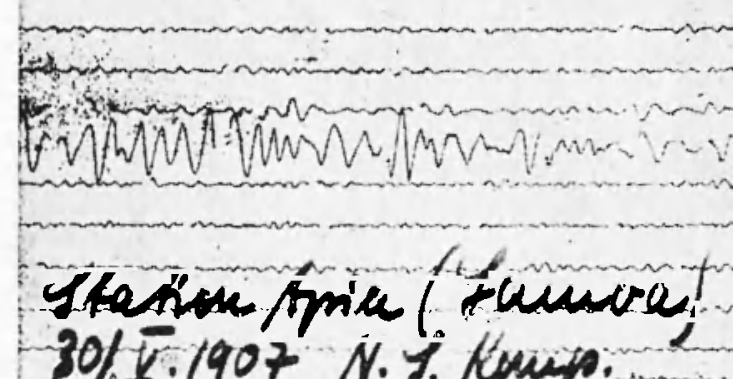

$30 \%$ F. 1907 N.T. Komp:

$\stackrel{\rightarrow}{\rightarrow}$ Elonithichts.

Fig. 17 . Horizontal-Seismogramme von Apia zum Erdbeben auf den Tonga-Plateau vom 31. Mai 1907. 
len Welle von $16.3 \mathrm{~km} / \mathrm{sec}$. Auch diese, lisher unhekannte Schicht ist, wie die Poisson' sche Zahl 0.30 (Fig. 13) zeigt, kristallin. Wiechert ${ }^{(36}$ ) loatte hereits im Jahre 1926 hei Fernsprengversuchen in den Aufzeichnungen des von ihm konstruierten Vertikal-Seismographen mit zweimillionenfacher Vergrösserung Reflexionen aus diesen tiefen Horizonten gefunden. Da die Sprengentfernung nur $18 \mathrm{~km}$ letrug, so ist hei dem felsigen Untergrund von Sprengstelle und Station eine Verwechslung mit Schichtwellen ausgeschlossen. Krumbach ( ${ }^{37}$ ) hercchnete die Tiefe einer reflcktierten Schicht zu $146 \mathrm{~km}$, henutzte daleei aher eine Wellengeschwindigkeit von nur maximal $8 \mathrm{~km} / \mathrm{sec}$.

In $183 \mathrm{~km}$ Tiefe scheint plastisches Material zu heginnen, wie aus Reflexionen an dieser Grenzfäche und aus anderen Anzeichen in den Seismogrammen geschlossen werden kann. Schweydar $\left({ }^{38}\right)$ herechnete aus dem Verlauf der Schwere in Küstengehiet die durchschuittliche Dicke der festen Erdkruste unter der Kontinenten zu etwa 200 Kilometer.

Die ausgezeichnete Uhereinstimmung der Beohachungsergelnnisse bei der Sprengung auf Helgoland mit den Resultaten der Untersuchungen von Caloi, Di Filippo \& Marcelli, Rosini und Valle an $P^{*}$ - und $P_{\mathrm{n}^{-}}$ Wellen von 5 italienischen Erdbehen geht aus der Figur 18 bervor.

Alles in allem hahen die seismischen Untersuchungen einen Aufhau der Erdkruste ergehen, mit dem sich Vorstellungen, wie Airy sie einmal hatte, nicht vertragen. W as für die Hypothese von Airy gilt, gilt auch für die Einknickungstheorie (buckling hypothesis) von $\mathrm{Ve}$ ning-Meinesz, $\left({ }^{30,40,41}\right.$, nach der die Erdkruste bei seitlichem Zusammenschul, der Schichten nach unten eingreknickt anstatt nach ohen aufgewölht werden soll. Vening-Meinesz erklärt Streifen von isostatischen negativen Schwereanomalien im Indischen Archipel, im Antillenlogen und an anderen von ihm untersuchten Stellen der Erd hzw. Meercsoberflache durch Sialwülste, die in das spezifisch schwerere Sima hinahgedrückt worden sein sollen. Seine Theorie unterscheidet sich demnach, wenigstens was ihre Auswirkung auf die Deutung von negativen Schwere-Anomalien betrifft, nicht wesentlich von der Hypothese von Airy. Infolgedessen gilt auch das dort hereits Gesagte, dass nämlich eine solche Einknickung schon deshalh nicht möglich ist, weil die erste naclogiehige (plastische) Schicht erst in $110 \mathrm{~km}$ Tiefe heginnt. Isostatiscl, reduzierten Schwerewerten kommt auch solange keine geologische Bedeutung $\mathrm{zu}$, wie der modus operating, wie Airy es ausgedriickt hat, unlsekannt bleiht. Anders verhält 
es sich mit der Bouguer-Anomalie, weil bei ilıer Berechnung nicht von einer vorgefassten Meinung über den modus operating ausgegangren wird.

In der Figur 19 ist die geographische Situation in Indischen Archipel sowie die Achse der negativen Anomalie nach Vening-Meinesz dargestellt. Ausserdem enthät der Lageplan den Verlauf vou zwei

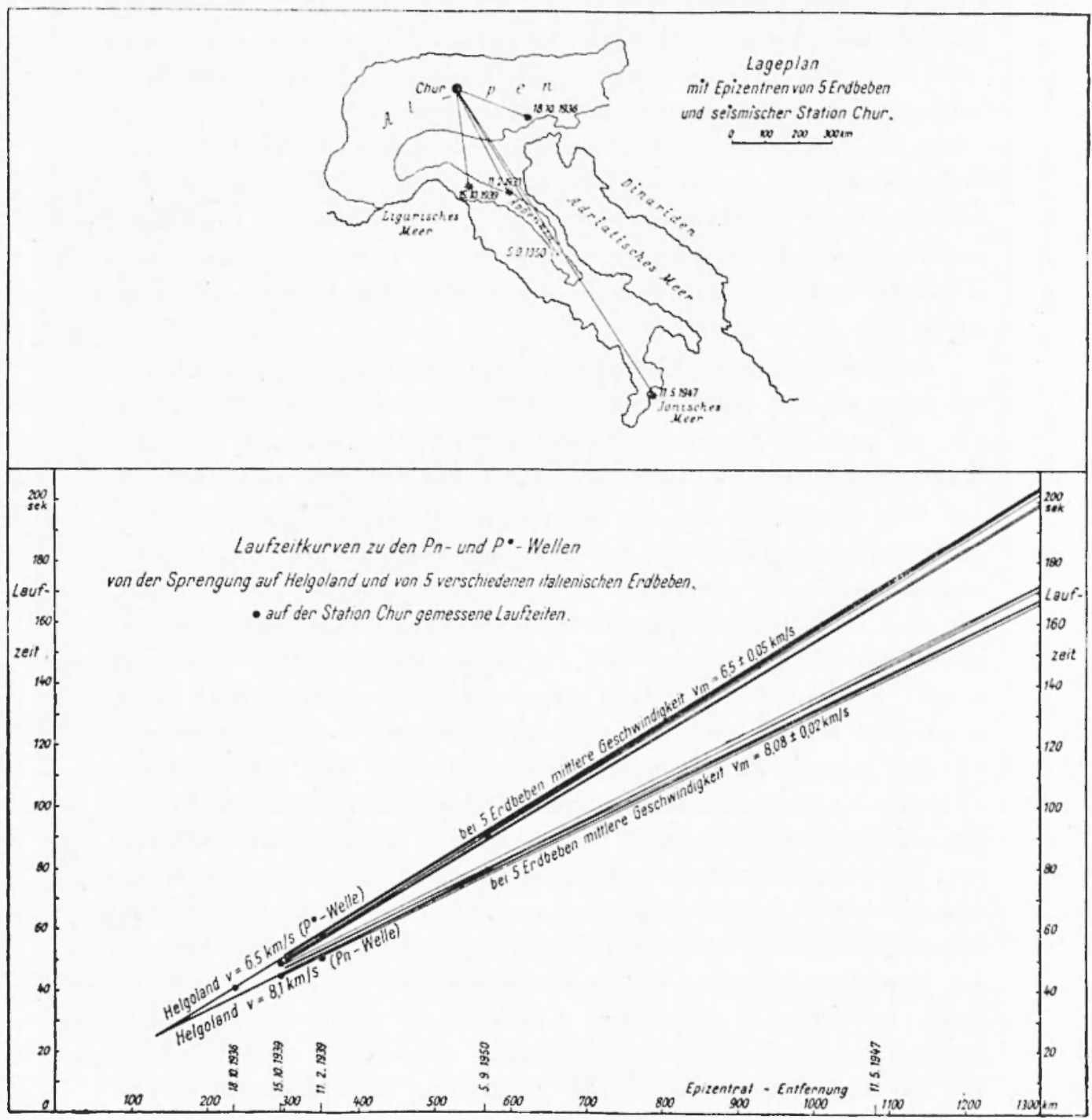

Fig. 18 - Lageplan mit Epizentren und Laufzeitkurven zu den $P^{x}$ und $P$..-Wellen bei der Sprengung auf Helgoland und bei 5 italienischen Erdbeben. 


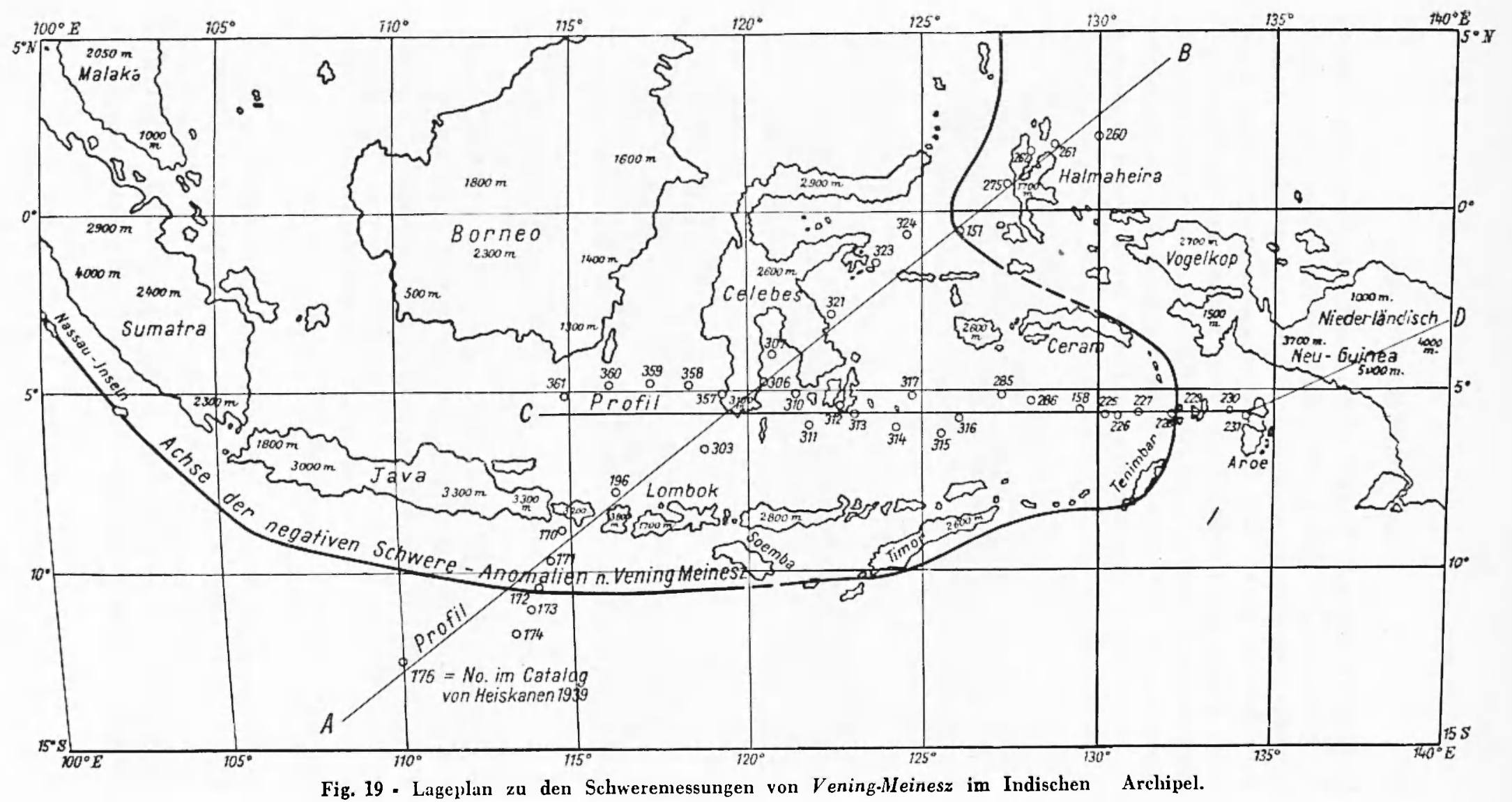


Profilen $A B$ und $C D$. Es fïllt zunïchst auf, dass die Achse links in der Figur mit der Achse der Sumatra vorgelagerten Nassau-Inseln zusammenfällt und sich im mittleren Teile mit der Achse suhmariner Aufwölbungen deckt. Alle Querprofile senkrecht zum Verlauf der Achse zeigen die gleiche Erscheinung, worauf Vening-Meinesz ${ }^{(11}$ ) selhst hereits hingewiesen hat. Das Minimum ist demnach auf diese Inseln hzw. auf die suhmarinen Rücken zurückzuführen. Die in den Figuren 20 und 21 wiedergegehenen Profile lassen diesen Zusammen-

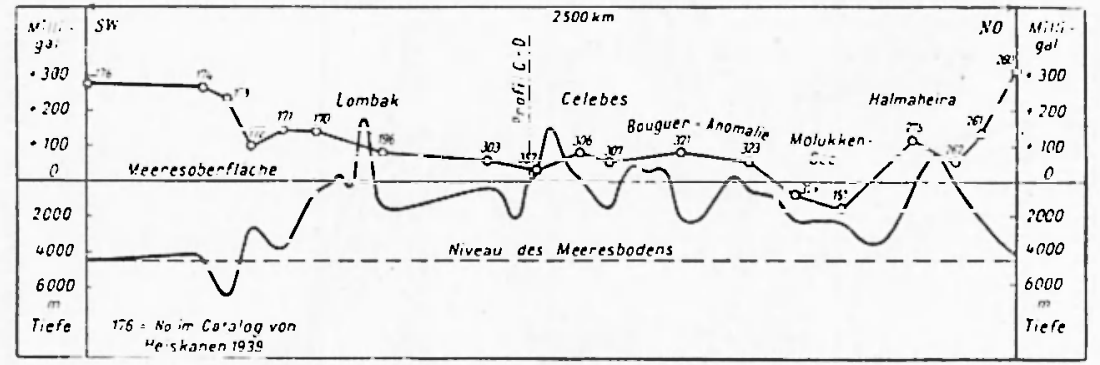

Fig. 20 - Profil der Bouguer.Schwere-Anomalien nach der Linie AB in der Figur 19.

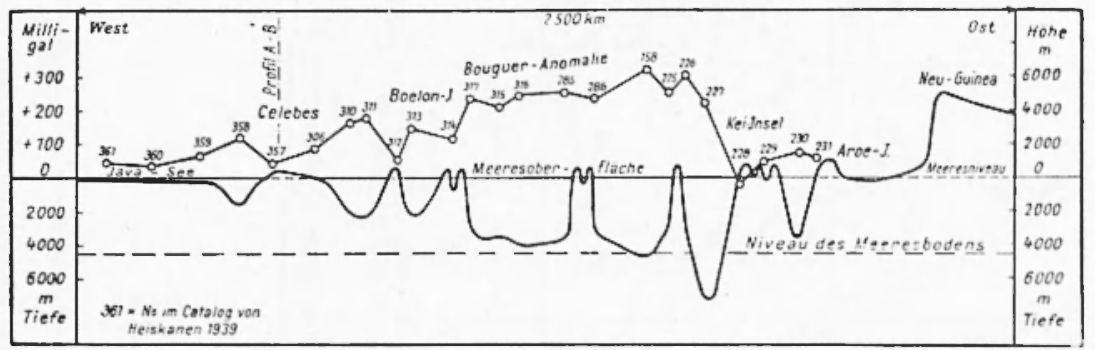

Fig. 21 - Profil der Bouguer-Schwere-Anomalien nach der Linie CD in der Figur 19.

hang klar erkennen. Zwischen ilmen und dem in der Figur 22 dargestellten Profil von Manila his Honolulu mit den Philippinen, den Inseln Yap und Guam sowie den Hawai-Inseln hesteht kein prinzipieller Unterschied. Es ist deshalh, wie auch Gerth (42) hei geologi-

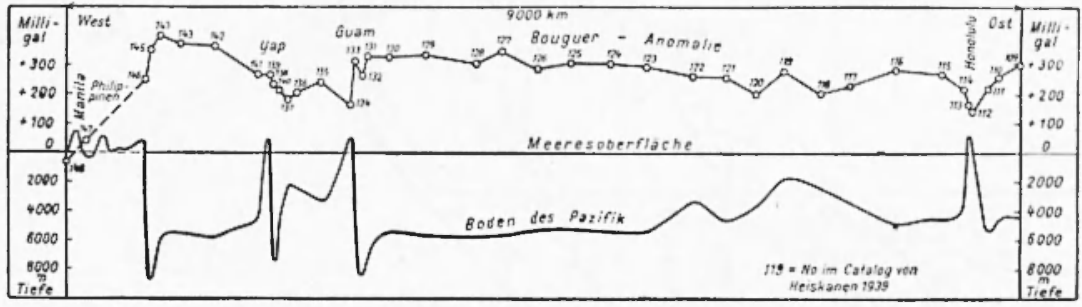

Fig. 22 - Profil de: Bouguer-Schwere-Anomalien von Manila bis Honolulu. 
schen Vergleichen feststellt, nicht ersichtlich, weshalb in Indischen Archipel ein Sonderfall angenommen wird, zumal auch das in der Figur 2 wiedergegehen Profil von den Alpen bis Sizilien in der Poelene cin ähnliches Minimum zeigt.

Die Einknickungstheorie von Vening-Meinesz schien in den Versuchsergebnissen scines Mitarbciters Kuenen ( ${ }^{43}$ ) eine Stütze gefunden zu haben, bei denen, wie die Figur 23 illustricrt, eine auf der Wasser-

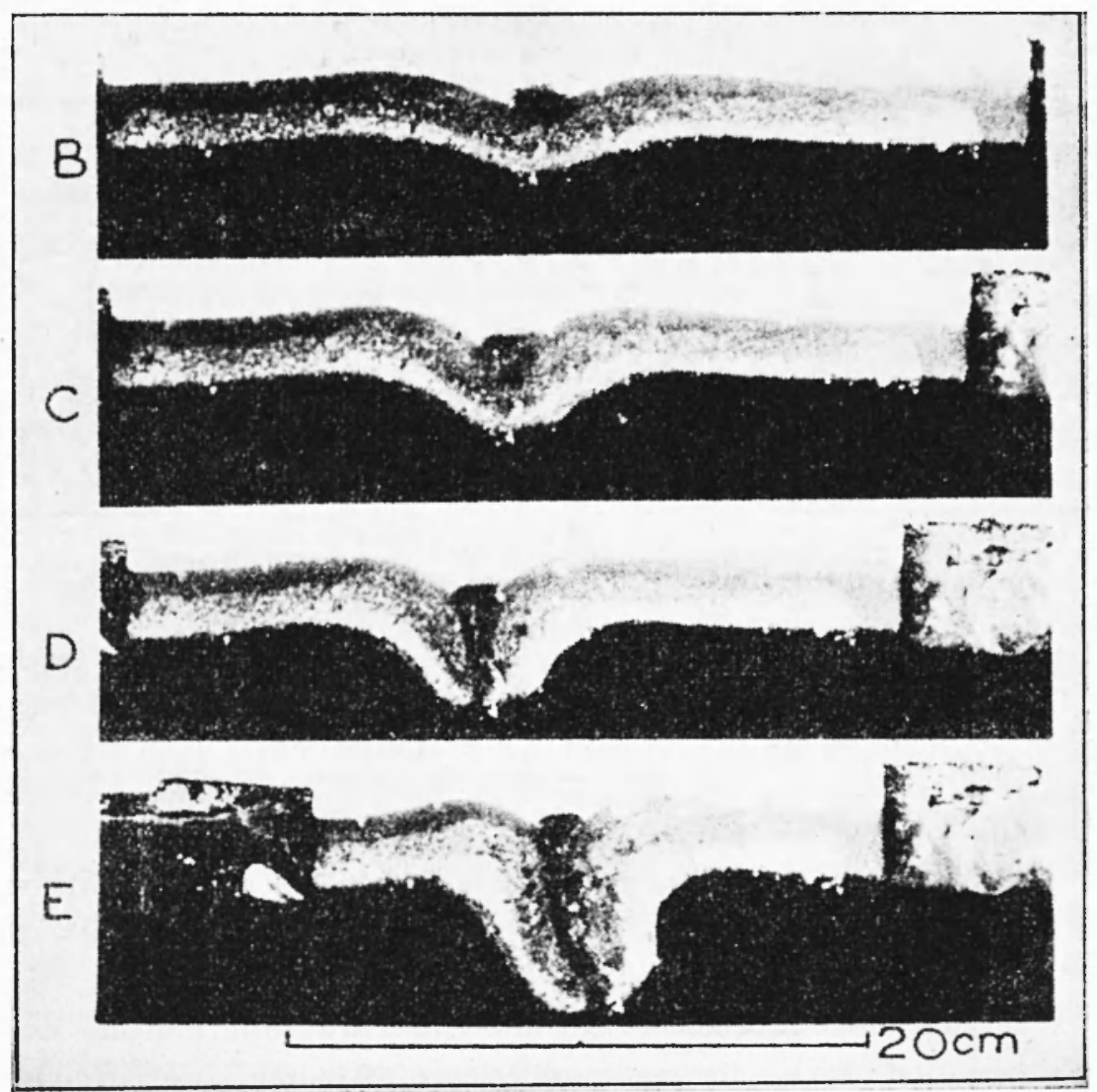

Fig. 23 - Modell-Versuch von Kuenen zur Einknickungstheorie von Vening-Meinesz.

oberfliche scbwimmende Paraffin-Schicht beim seitlichen Zusammenschieben nach unten eingeknickt wird. Nach Griggs $\left({ }^{44}\right)$ stellte sich diese Einknickung nach unten im allgemeinen aber nur dann ein, wenn die Knickstelle vorher etwas belastet worden war. Während VeningMeinesz den Widerstand des plastischen Simas in seinen Berechnungen 


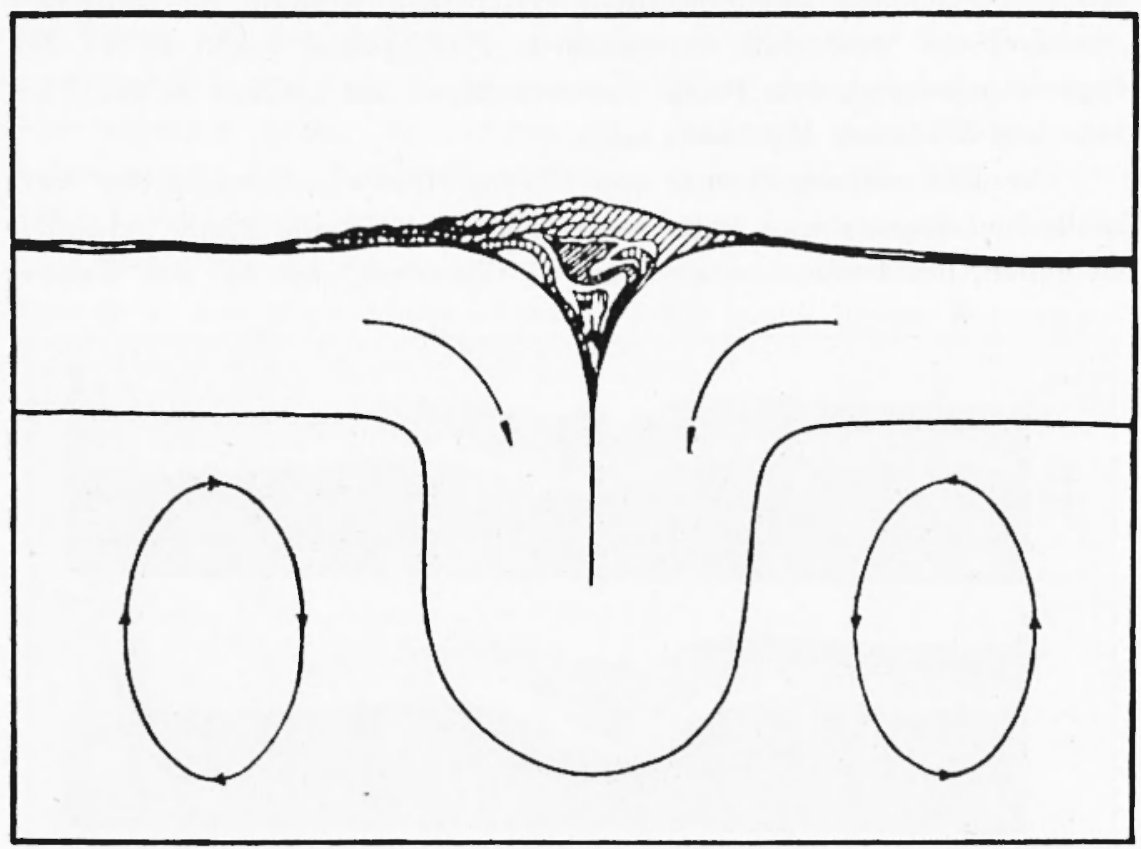

Fig. 24 - Profil zur Convections-Theorie von Vening-Meinesz.

vernachlïssigen zu können glaul,t, wies Griggs experimentell nach, dass keine Einknickung nach unten, sondern eine Verdickung nach ohen stattfand, wenn die Viscosititt des Sulstratums "vergleichl)ar" mit den wirklichen Verhältnissen in der Erde gemacht wurde. Tromp ( ${ }^{45}$ ), der wie Griggs sehr eingehend auf die Ansichten von Vening-Meinesz eingeht, komnt in seinem sehr lesenswerten Buche, dem auch ein ausführliches Literatur-Verzeichnis beigefügt ist, zu dem gleichen negativen Ergehnis.

Bei der ausgesprochenen Schichtung der Erdkruste und des Erdmantels sind auch die von zahlreichen Autoren, wie Holmes, Griggs, Pekeris, Vening-Meinesz u.a., unter der Voraussetzung eines nicht geschichteten Erdmantels theoretisch behandelten Strömungen, wie sie die Figur 24 zeigt, nicht möglich. Griggs rechnete mit dieser Unmögliehkeit, wie aus folgenden Worten hervorgeht: "The discontinuities within the crust are not considered here hecause it is thaught that they act only as secondary modifying influences on the reaction of the crust to the subcrustal convection. It is entirely possible that this 
ist erroneous, and that the crustal discontinuities play a fundamental role $)$.

Wenn auch geothernnische Strömungen in der Art, wie sie die Figur 24 darstellt, nicht möglich sind, so kommt doch der Geothermik fïr die Erklärung der Verteilung von Wasser und Land sowie der Hebungen und Senkungen der Erdoherfläche eine sehr wesentliche Bedeutung zu. Boscovich ( ${ }^{47}$ ) schrieh schon im fünften Buche seines zusammen mit Maire herausgegebenen grossen Werkes, das 1755 in Rom in lateinischer und 1770 in Paris in französischer Sprache erschienen ist: "Mais les montagnes se forment, je pense, pour la plupart par l'effet d'une chaleur interne qui soulève les couches de la terre les plus proches de la surface; et s'il en est ainsi, cette élévation n'ajoute aucune nouvelle matière, et le vuide renfernı́ dans l'intérieur de la montagne compense la masse qui le couvre ).

C. Babbage (48 )gah im Jahre 1847 der Ansicht Ausdruck, dass die allmählichen Hebungen und Senkungen der Erdoherfläche lediglich eine Folge der durch Erwärmung oder Ahkühlung verursachten Ausdehnung oder Zusammenziehung tieferer Erdschichten seien. Er zeichnete bereits geothermische Profile durch Kontinente und Orcane sowie die Ânderungren dieser Profile durch Denudation und Sedimentation. Von den zahlreichen Autoren, die Babbage's Anschanungen vertreten, sei der damalige Präsident der Liverpool Geological Society, T. Mellard Reade (19), besonders hervorgehohen, weil er sein inı Jahre 1886 erschicnenes, umfangreiches und äusserst lesenswertes Buch üher die Entstehung ron Gebirgszïgen fast ausschliesslich geothermischen Darlegungen gewidmet hat. Reade glaubt sogar olıne plastische Zwischenschicht auskommen zu können, die er deshalh nicht für wahrscheinlich hält, weil die darüberliegenden festen Schichten der Erdkruste dann noch selır viel mobiler wären als sie es ohnehin schon sind.

Orstrand ("50) untersuchte den Verlauf der Isothermen in Gebirgen und fand, dass sie bis etwa loo $\mathrm{km}$ Tiefe konvex nach oben gekrimmt seien.

Nach der Entdeckung der plastischen Zwischenschicht und bei den seit dem Erscheinen des Buches von Reade gewonnenen Erkenntnissen über die Wärme-Erzeugung beim Zerfall radioaktiver Elemente in der Erdkruste erscheint es zweckmässig, auf die Bedeutung der 
Geothermik für die Erklärung der Konfiguration der Erdoberflïche nähereinzugehen.

Die Figur 25 zeigt die ausserordentlich grossen lokalen und regrionalen Unterschiede in geothermischen Tiefenstufen, die zwischen einigen und mehr als hundert Metern schwanken. Selbst in einer kleinen geologiscben Struktur können die Unterschiede sehr hedeutend

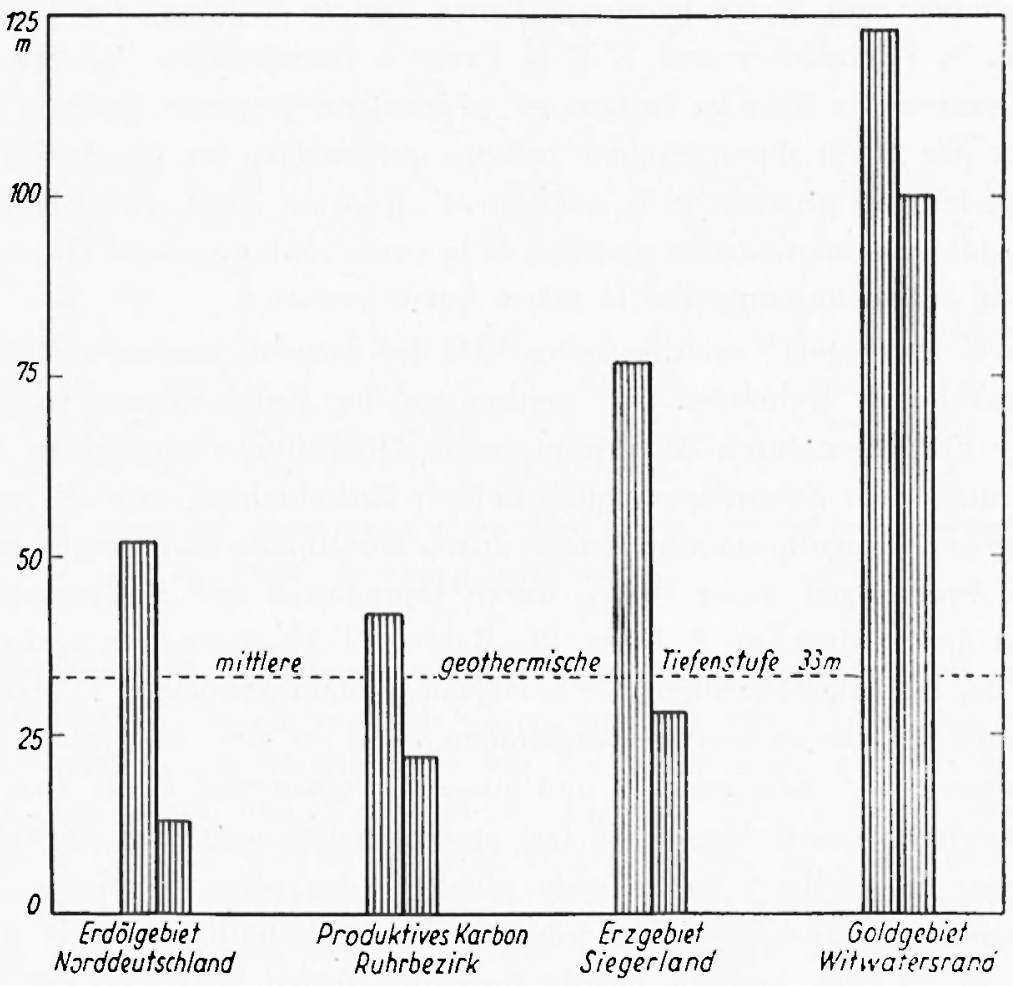

Fig. 25 - Verschiedene geothermische Tiefenstufen.

sein (Fir. 36). Nach der von Jeffreys ( ${ }^{11}$ ) aufgestellten Temperaturkurve für das Erdinnere (Figr. 27) nimmt die geothermischc Tiefenstufe mit der Abnahme des Gehaltes der Gesteine an Uran, Thorium und Kalium zu und beträgt in $100 \mathrm{~km}$ Tiefe bereits $175 \mathrm{~m}$. Eingehende Untersuchungen über die Radioaktivität der Erde sind in neuerer Zeit von Festa ( $\left.{ }^{52}\right)$ und Santangelo (52) durchgeführt worden.

In den Meeren nimmt die Temperatur von der Oberfläche bis zum 


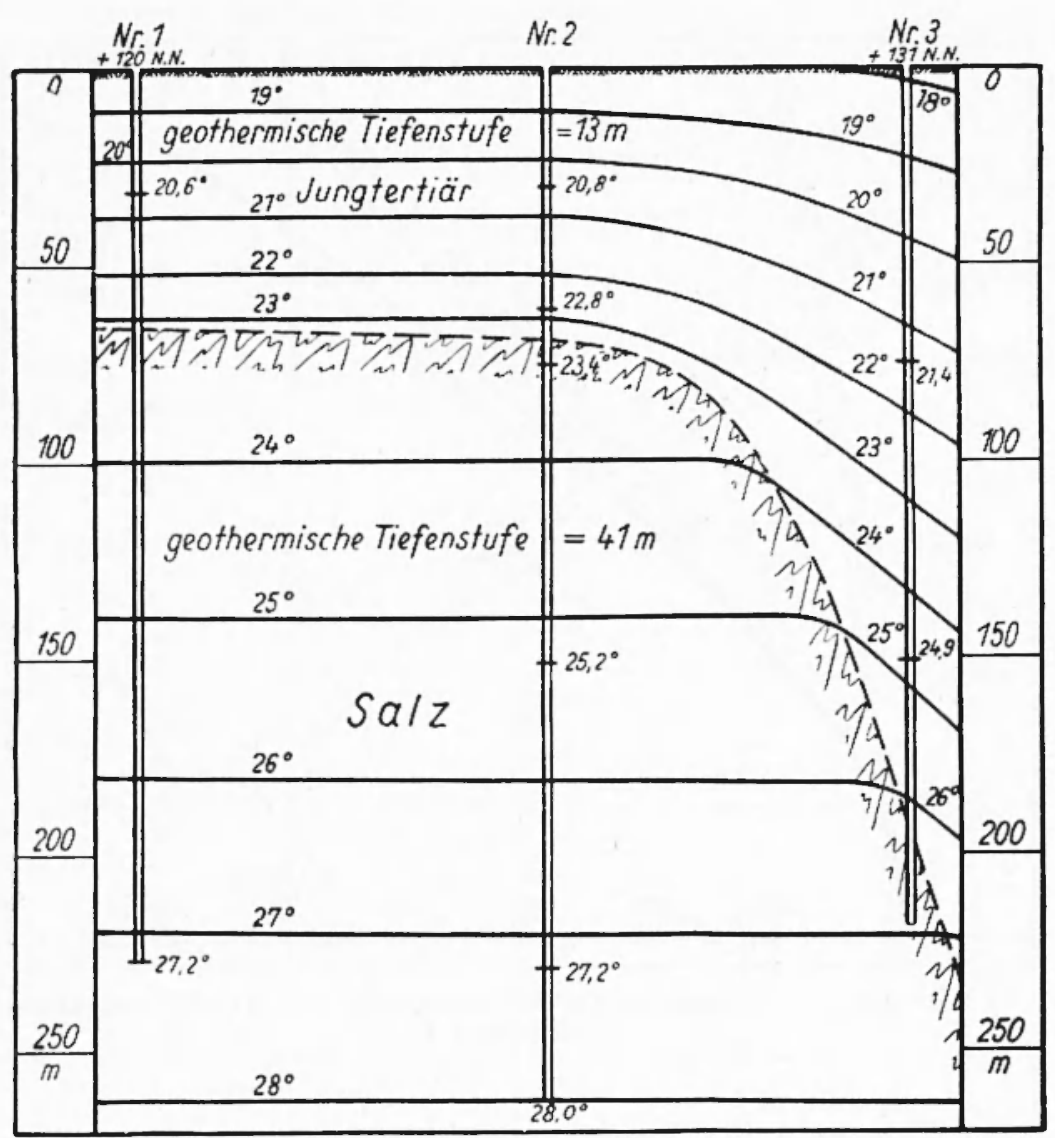

Fig. 26 - Isothermen im Profil durch eine geologische Struktur von Halbouty.

Meereshoden nicht zu, sondern his auf fast $0^{\circ}$ ab (Fig. 28 links). Auf der Schwedischen Alhatros'Expedition 1947/48 sind in einer Ozeantiefe von $7.992 \mathrm{~m} \mathrm{nur}+11 / 2 \mathrm{Grad}$ Celsius gemessen worden.

Wird num die Jeffrey'sche Temperaturkurve, wie es in der Figur 29 geschehen ist, cinmal von der Olerffäche des Festlandes und das andere Mal von der Meeresoberflïche aus nach unten aufgetragen, so

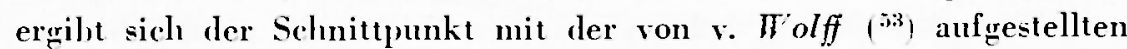
Schmelzkurve cines hestimmten Tiefengesteines unter dem Mcere erhelhlich tiefer als unter dem Festlande. Die kristallinen Schichten sind also unter den Ozeanen dicker als unter den Kontinenten, so dass sich das Phänomen der gleichen Schwere auf dem Festlande und auf dem Mecre zum teil hierdurch erklären lässt. 


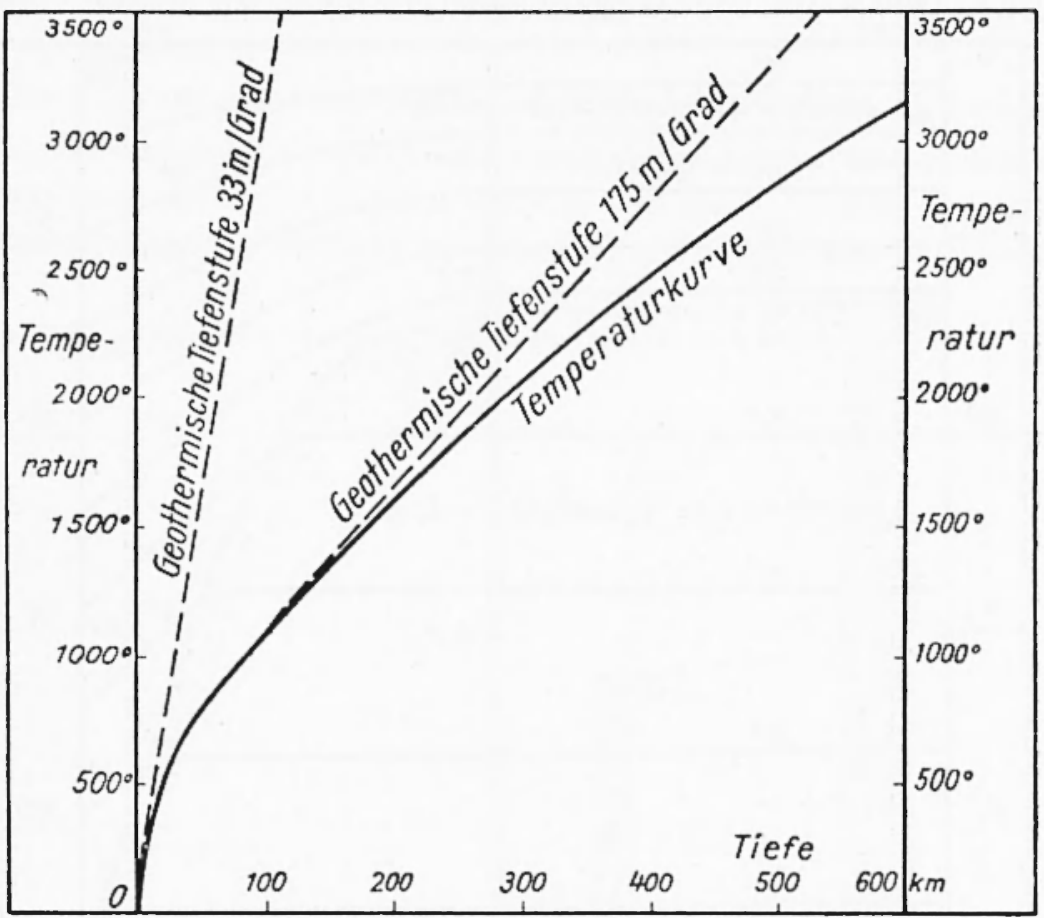

Fig. 27 - Zunahme der Temperatur im Erdinnern, nach Jeffreys und geothermische Tiefenstufen.

Die Ergelonisse der Temperaturmessungen in den Alpentunneln lassen den Schluss zu, dass im Meeresniveau unter dem Gebirge ein Temperaturunterschied gegenïl)er dem gleichen Niveau des Festlandes von etwa $+100^{\prime \prime}$ besteht. Infolgedessen liegt natürlich auch die plastische Zwischenschicht unter den Alpen höher als unter ilıren Flanken. Nach Berechnungen von Rosenbach ( ${ }^{(j)}$ ) erzeugt dic im Profil der Figur 30 dargestellte Verdickung der plastischen Schicht genau die aus den Schweremessungen abgeleiteten negativen BouguerAnomalien.

Die "Aufwöllbung " der plastischen Schicht ist natürlich keine "Antiklinale " im gewönlichen greologischen Sinne, sondern nur eine Folge der durch die Pfeile in der Figur 30 angedeuteten Expansion von plastisch gewordenem Kristallin. Eine solche Volumenvermehrung wirkt sich besonders stark nach oben aus, weil eine Expansion nach unten ülserhaupt nicht und nach den Seiten hin nur in sehr beschränk- 


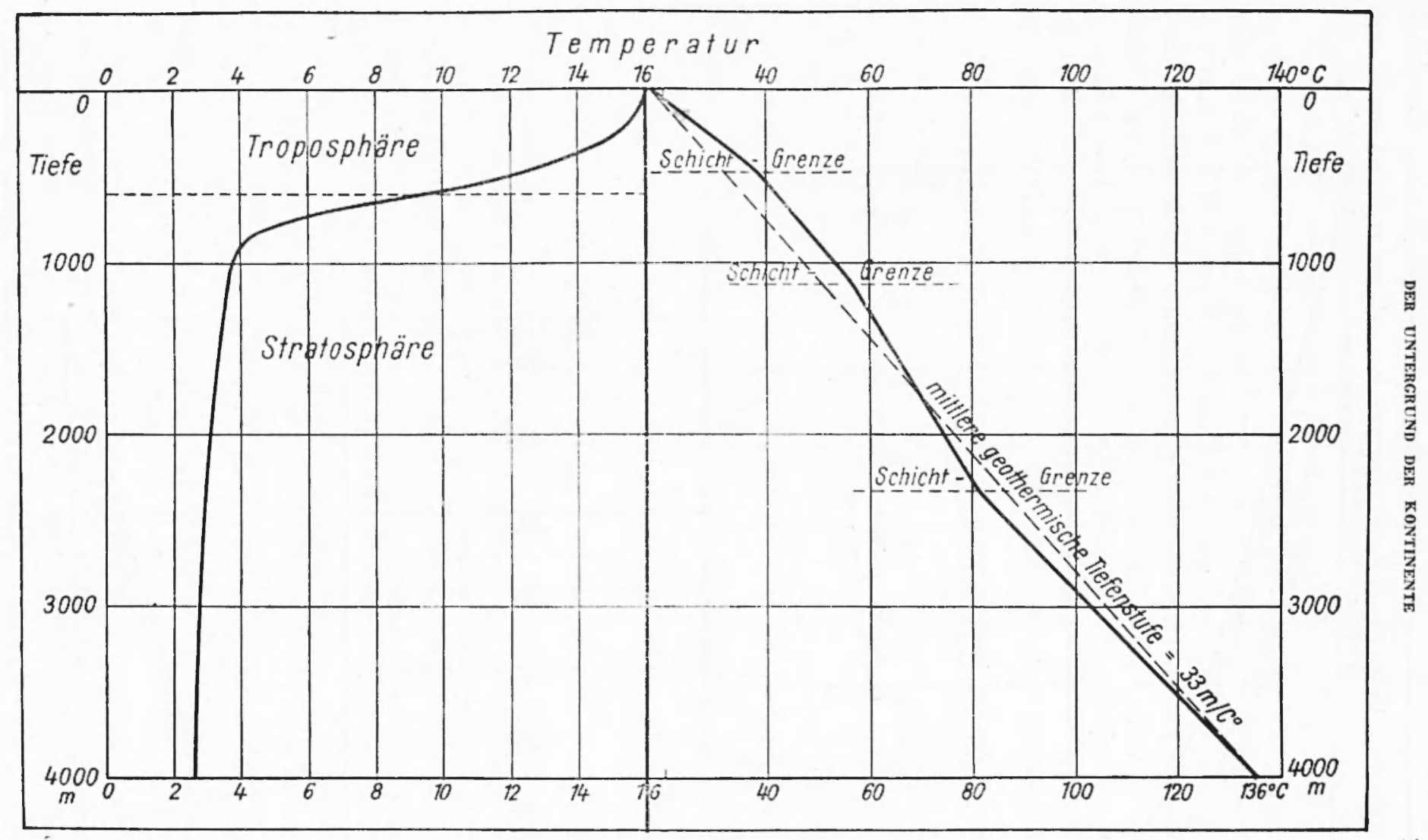

28 - Temperatur-Abuahme unter der Meeresoberfläche nach Defant und-Zmulmme unter der Oberflache des Festlandes (schematisch), 


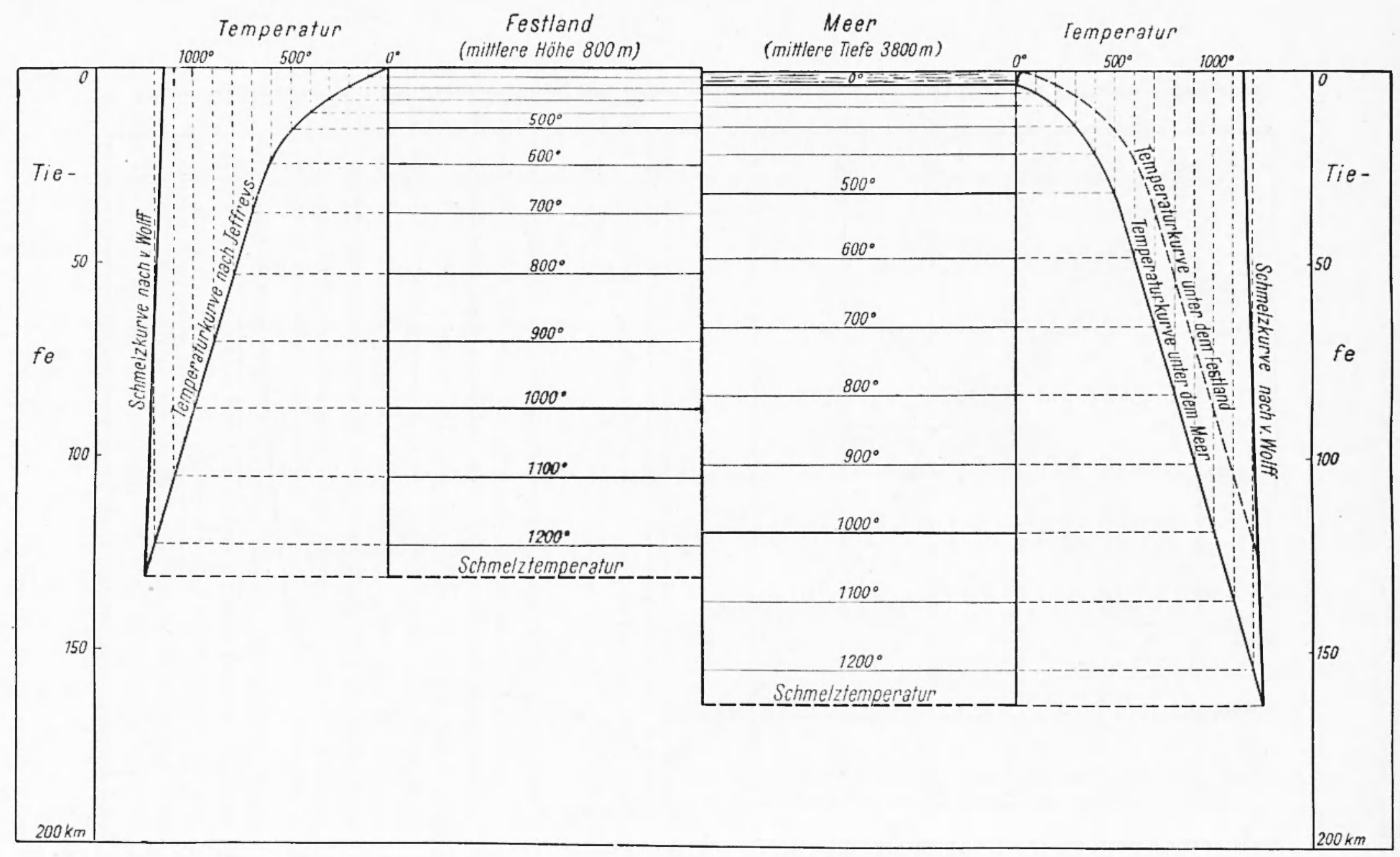

Fig. 29 - Geothermisches Profil durch die Erdkruste unter Festland und Meer sowie Temperaturkurve von Jeffreys und Sehmelzkurve von $v$. $W$ olff. 
tem Masse möglich ist. Die Gehirgszüge verraten demnach Verdickungen der plastischen Zwischenschicht.

Bischof $\left({ }^{55}\right)$, damats Professor der Chemie an der Universität Bonn, zeichnete bereits in Jahre 1838 die Oberflache des Magmas parallel zum Profil der festen Erdolıerfäche. Deely (56) bemerkt bei Erwạ̈ungen über den Bau des Kanadischen Schildes, dass Kompression und Faltung allein für die Gelirgshildung nicht ausreichend sind, in erster Linie komme die Ilelung infolge Ausdehnung hohe Temperatur aufweisender Tiefenschichten in Betracht. Hopkins ( ${ }^{i 7}$ ) schrieh hereits im Jahre 1836: “I suppose this clevatory force, whatever be its origin act upon the lower surface of the upliftet mass through the medium

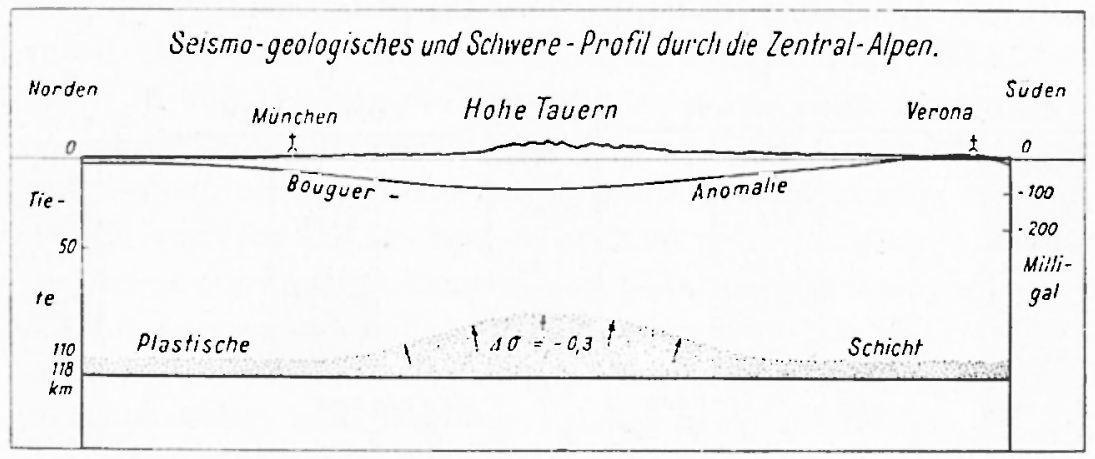

Fig. 30.

of some fluid, which may he conceived to he an elastic vapour or in other cases a matter in a state of fusion from heat m. Reid ("), seinerzeit Professor der Geologie an der John Hopkins University in Baltimore, der auclı als Seismologe hekannt geworden ist und an den Congressen der International Association of Seismologists teilnahm, ausserte sich zur Frage der Isostasie mit folgenden Worten: "The theory of isostasy tells us, quite definitely, that the elevation of mountains or the depression of the ozeans must he due to vertical forces hrought about hy a decrease or increase of the material under these regions ". Clarence Eduard Dutton ("), der für den Lastenausgleich in der Erde das Wort "Isostasy " geprïgt hat, führte am 27. April 1889 in seinem Vortrage vor der Philosophical Society of Washington aus: "Wathever may have heen the cause of the great regional uplifts, it in no manner, affects the law of isostasy. What the real nature of the uplifting force may he is, to my mind, an entire mystery; hut, 
I think we may discern at least one of its attribute, and that is a gradual expansion or diminuation of the density of the subterraneum magmas. If the isostatic force is operative at all, this expansion is a rigorous consequence, for whenever a rise of the land has taken place on of two things has happened: the region aflected has either gained an accesion of mass or a mere increase of volume without increase of mass. Hence, I infer, that the cause which elevates the land involves an expansion of the underlaying magmas, and the cause which depresses it is a shrinkage of the magmas. The nature of the process is, at present, a complete mystery ".

Wie das Profil durch Kontinente und Ozeane in der Figur 31

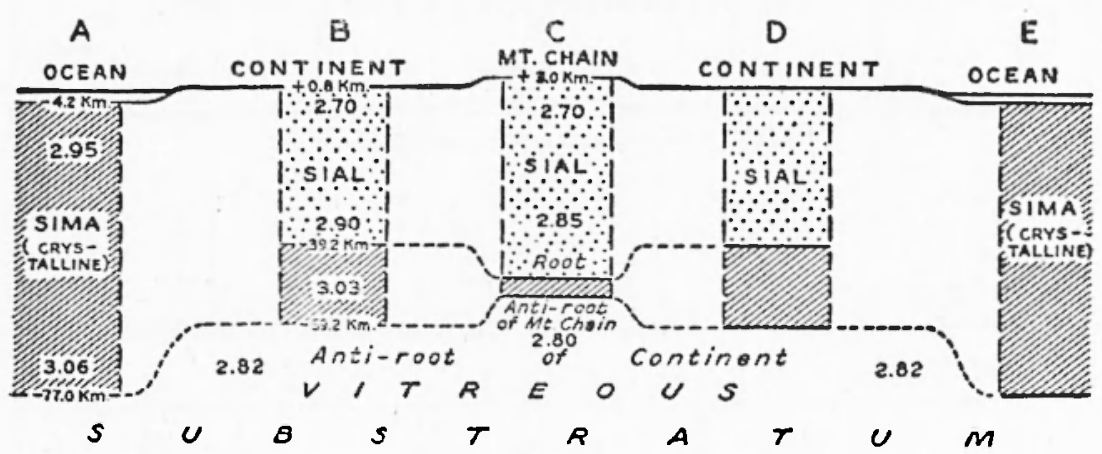

Fig. 31 - Geologisches Pronl durch Continente und Ozeane nach Daly.

zeigt, entsprechen die Ergebnisse der in dieser Abhandlung geschilderten Untersuchungen des Verfassers der bereits in Jahre 1938 in dem beriihmten Buche "Architecture of the Earth ) des GeologieProfessors an der Harvard-Universität, R.A. Daly, vertretenen Ansicht: Wurzel (root) unter dem Meere, Gegenuurzel (anti-root) unter dem Festland, insbesondere auch unter den Gebirgen. Diese Aussage ist das Gegenteil von dem, was zahlreiche Geologen und Geophysiker bisher angenommen haben.

Wie die Seismik bewiesen hat, dass die Ilypothese von Airy unhaltbar ist und die Gebirge keine Wurzel haben, so ist sie auch in der Lage, die Verdickungen der plastisehen Zwischenschicht zu bestimmen und damit die Voraussetzung für eine hypothesenfreie isostatische Schwere-Reduction zu schaffen. Das masstabliche Profil in 
der Figur 32, in dem der $5 \mathrm{~km}$ tiefe Pazific und die $4 \mathrm{~km}$ hohen Rocky Mountains nur durch kleine, kaum sichthare Verdickungen der die Erdolserfläche darstellenden Linie zum Ausdruck kommen, zeigt, dass es sich nur um eine Aufgalee der Sprengseismik handeln kann. Bei dem heutigen Stande der experimentellen Seismik wird die Lö-

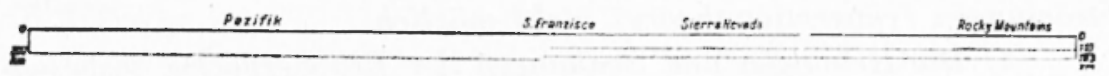

Fig. 32. - Masstabliches Profil durch dic Rocky Mountains und den astlichen Pazific mit plastischen Schichten in tiefen Üntergrunde.

sung des Problems nicht auf unüherwindliche Schwierigkeiten stossen, zumal die 8- und $11 \mathrm{~km} / \mathrm{sec}$-Schichten, wie aus den Profilen der Figuren 12 und 14 sowie den Beolyachtungen von Angenheisler an pazifischen Erdlsehen hervorgeht, geschlossene Gürtel um die Erde bilden und deshall, als seismisclee Leithorizonte henutzt werden können.

Es wäre für die im Septemlyer 1950 unter italienischer Führung in Verona gegründete "Organisation Séismologique Européenne" und die vor kurzem auf holländische Anregung hin im Haag ins Leben gerufene "European Association of Exploration Geophysicists " eine wissenschaftlich und praktisch gleich lohnende Aufgalse, die Dicke der plastischen Zwischenschicht im Alpengeliet sprengseismisch zu ermitteln. Mit der Lösung dieser Aufgabe würde dem 200-jührigen Rätselraten um die Ursache des Massendefizits unter den Hochgelirgen ein Ende gemacht werden. Da die Geologie in aller Welt ein grosses Interesse an der Lösung des Problems der Gebirgsbildung hat, werden die Staaten gern bereit sein, die für die Durchführung der sprengseismischen Untersuchungen erforderlichen Mittcl zur Verfügung zu stellen.

\section{ZUS.AMMENFASSUNG}

De Ergebnisse der Untersuchungen sind folgende:

1) Die Schwimmhypothese von Airy, wie sie von vielen spateren Autoren verstanden worden ist, verträgt sich nicht mit seismi. schen Beobachtungsergebnissen. Die Sierra Nevada und die Alpen haben keine Turzel (root), es sind vielmehr Anzeichen einer Gegenuur. zel vorlanden.

2) Die Einhnichungstheorie (buchling-hypothesis) von Vening- 
Mejnesz ist nicht haltbar und zur Erhlärung der Streifen negativer Schwere-Anomalien im Indischen Archipel auch nicht erforderlich.

3) Bei der seismisch festgestellten Schichtung der Frdhruste und des Erdmantels sind die von zahlreichen Autoren, wie Griggs, Holmes, Pekeris, Veninw-Meinesz und anderen unter der Voraussetzung eines nicht geschichteten Erdmantels theoretisch behandelten Strömungen (convection-theory) nicht möglich.

4) Die Hebungen und Senkungen der Erdoberfläche, insbesondere auch die Heraushebung der Gebirge, wird in Ubereinstimmung mit den Ansichten von Boscovich (1755), Hopkins (1836), Bischof (1838), Reade (1886), Dutton (1889), Decly (1910), Reid (1911), Tromp (19.37) und anderen. Autoren durch geothermisch bedingte Volumen-Anderungen von Tiefenschichten erhlärt.

5) Die kristalline Erdliruste ist unter den Meeren dicker als unter dem Festlande (roots und anti-roots von Daly 19.38).

6) Es sind sprengseismische Untersnchungen erforderlich zur Ermittlung der Dicken der plastischen Tiefenschichten unter dem flachen Lande und unter den Gebirgen.

\section{SLIUIRY}

The results of the investigations are the following:

1) The swim-hypothesis of Airy, as conccived by many authors, is not in accordance with seismic observations. The Sierra Nevada and The Alps have no root, but there are indications of anti-roots under the mountains.

2) The buckling-hypothesis of Vening-Meinesz camnot be maintained and is not necessary for the explanation of the stripes of negative gravity-anomalies in the Indian Archipel.

3) In view of the fact that the earth's crust and mantel, according to seismic studies, show a pronounced stratification, the convection-theory of Griggs, Holmes, Pekeris, Vening-Meinesz and other anthors cannot be maintained.

4) The elevations and depressions of the surface of the earth, especially the uprise of the countains are explained by geothermically caused variations of the volume of deep seated materials. This view is accordance with the opinion of Boscovich (1755), Hopkins (1836), Bischof (1838), Read (1886), Dutton (1889), Deely (1910), Reid (1911), Tromp (1937) and other authors. 
5) The crystalline crust of the earth is thicker under the oceans than under the continents (roots and anti-roots of Daly, 1938).

6) In order to determine the thickness of the plastic layers within the earth's crust seismic experiments uith artificial earth-quakes (blastings) should be made uithin, and outside of, the mountuinranges.

\section{BIBLIOGRAFIA}

(1) Inziskanex W.: Catalogue of the isostatically reduced gravity stations. Ann. Acad. Scientiarum Fennicae. Ser. A. Tom. LI, N. 10, Helsinki 1939.

( $\rightarrow$ MontLl. C.: La rete geofisica e geodetica in Italia nel sto stato attuale e nei suoi rapporti con la struttura geologica superficiale e profonda. Pubblicatione N. 121 dell'Istituto Nazionale di Geofisica. Trieste 1946, p. 1.77.

(3) Pнатr J. H.: On the attraction of the Himalaya Mountains and of the elevated regions beyond them upon the plumb-line in India. Phil. Trans. Royal Soc London, vol. 1.45, p. 51, 1855.

(4) Ainy G. B.: On the computation of the effect of the attraction of mountain masses as disturbing the apparcm astronomical latitude of stations in geodetic sterveys. Phil. Tran. Royal Soc. London, vol. 115. p. 101, 1855.

(5) Niftumugr Tu.: Die Schuerebestimmungen der schueizerischen geodä. tischen Commission und ihre Frgebnisse. Verhandlungen der Schweizerischen Naturforschenden Gesellschaft, Schaffhausen, $192 \overline{1}$.

(b) Hayom J. F.: The figure of the Earth and isosfasy from measurements in the Viniled States. Washington 1909, p. 1-176.

(i) Pratr J. H.: On the deflection of the plumb.line in India, caused by the attraction of the IHimalaya Mountains and of the sletated regions beyond. and its modification by the compensating effect of a deficiency of matter below the mountain mass. Phil. Trans. Royal Soc. London, vol. 149, p. 745-778, 1859.

(8) Sunvax E.: Ober die Erdhrustendicle und die isostatische Compensation der Schweizer Alpen. Ann. Acad. Scientiarum Finnicae. Ser. A., Tome XXXVII, Helsinki 1933, p. 1-27.

(9) Heiskaves W.: Tintersuchungen über Schwerkraft und Isostasie. Suomen Geodetissen Laikoksen Julkiisuj:ı, N. t, p. 1-96. Melsinki 1924.

(10) Horopanex P. E.: On the gratity field and the isostatic structure of the Earlh's crust in the East Alps. Ann. Acad. Scientiarum Finnicae. Ser. A. III. Geologiea-Geographica 12, p. 1.9.1, Helsinki 19.17.

(11) Gutribeng B.: Seismological evidence for roots of mountains. Bull. Geol. Soc. Americi, vol. 54, p. 473-198, 1943.

(19) Waniner E.: Ober den Tiefgang der Alpenfallung. Edogae Geologicae Helvetiae, vol. 41, p. $125-134,1948$.

(13) Calou P.: Ricerche su terremoti ad origine vicina. Scosse del Consiglio dellotlobre 1936-XI\%. Pubblicazione dell'Istituto Nazionale di Geofisical del Consiglio Nazionale delle Rirerche. N. 7, p. 1.11, Roma 19.16.18. 
(14) -: Caratteristiche sismiche dell Appennino tosco-romagnolo. Pubblicazione dell'Istituto Nazionale di Geofisica del Consiglio Nazionale delle Ricerche. N. 36, p. 1-15, Roma 1918-50.

(15) Di Filippo D. - Marcelli L.: Uno studio sul terremoto del Gran Sasso d'talia del 5 settembre 1950. Annali di Geofisica, vol. IV, N. 2, aprile 1951, p. $213-239$.

(10) -: Tempi di tragitto delle onde $P^{*}$ e spessore dello struto del granito nellttalia Centrale. Annali di Geofisica, vol. IV, N. t, ottobre 1951, p. 579-589.

(17) RosiNı E.: Il terremoto della Garfagna del 15 oltobre 1939.XYl1. Pubblicazioni dell'Istituto Nazionale di Geofisica del Consiglio Nazionale delle Ricerche, N. $4-1$, p. $1 \cdot 2-4$, Roma 1948.

(1S) Valle P. E.: Contributo allo studio delle caratteristiche sismiche del Mediterraneo centro-orientale. Pubblicazioni dell'Istituto Nazionale di Geofisica del Consiglio Nazionale delle Ricerche, N. 1+10, p. 1-16, Roma 19.18.

(19) Byerly P.: The first preliminary wates of the Nevada Farthquake of December 20, 1932. Bull. Seism. Soc. America, vol. 25, N. 1, January 1935, p. 62-80.

(-0) -: The Sierra Nevada in the light of isostasy (discussion). Bull. Geol. Soc. America, vol. 48, 1937, p. 2025-2031.

(21) -: Near Earthquates in Central California. Bull. Scism. Soc. America, vol. 29, N. 3, July 1939, p. 427-162.

(a) Mivtrop L.: Die Gliederung der Erdrinde und des Erdmantels nach seismischen Beobnchtungen. Nachrichten Ges. Wiss. Göttingen, math.-phys. Klasse 1947.

(23) - : On the stratification of the Earth's crust according to seismic studies or a large explosion and of earthquakes. Geophysies, vol. XIV, N. 3, July 1949, p. 321.336 .

(21) Angenheister G.: Discussionsbemerkung. Comptes Rendus XVIe Session Congrès Géologique International, 1926, p. 1507, Madrid 1928.

(25) Stoneley R.: Some new earthquakes reported in the International Seismological Summary. Monthly Notices, Geophysical Supplement, January 1931.

(26) Wiechfre E.: Über die Massenverteilung im Innern der Erde. Nachr. Ges. Wiss. Gottingen, math.-phys. Klasse 1897, Heft 3, p. 221.213.

(27) Daty R. A.: Architecture of the Earth. Newyork \& London 1938, p. 1.201.

(28) - : Strength and Structure of the Earth. New York 1910, p. 1-134.

(29) Моновоvicic St.: Das Erdinnere. Zeitschr. fïr Angewandte Geophysik, Band 1, Heft 2, 1925, p. 330 .

(30) Doi.ttin C.: Zur Physik des Fulkanismus. Sitzungsberichte Wiener Acad. Wiss. mathem.-naturw. Klasse, vol. CXII, Abt. 1, 1903, p. 681.

(31) Helniert F. R.: Die Tiefe der Ausgleichsfläche bei der Pratt'schen Hypothese für das Gleichgewicht der Erdhruste und der Verlauf der Schwerestorung vom Innern der Kontinente und Ozeane nach den Küsten. Sitzungsberichte Acad. Wiss. Berlin 1909, vol. XLVIII, p. 1192-1198.

(32) Bons A.: Schweremessungen und Isostasie. Berlin 1923. 160 Seiten, 31 figure.

(33) Gutenberg. B.: Variations in physical properties within the Earth's crustal layers. Am. Journ. Science, 243 A, Daly volume 1945, p. 312.

(34) BeNNonf H.: Uber die Art der Fortpflanzung der Erdbebenwellen im 
Erdinnern. Mitt. Erdbebencommission Acad. Wiss. Wien, Neue Folge N. XXIX, 1905.

(35) Anenueister G.: Beobachtungen an pazifischen Beben. Naclirichten Ges. $W^{\prime}$ iss. Göttingen, math.phys. Klasse 1921.

(30) Wiechert E.: Untersuchungen der Erdrinde mit Hilje von Sprengungen. Geol. Rundschau, vol. XVII, Heft 5, 1926, p. 339-346.

(37) Kоммвтси G.: Uber die Laufzeiten der Vorlaufer-und Wechselwellen bei Erdbeben in ihrer Beziehung zur Schichtung der Erdrinde. Verölf. Reichsanstalt fïr Erdbebenforschung, Jena 1926, Heft 5.

(38) Scuwt:Yoar W.: Uber Isostasie. Zeitschr. für Geophysik, II. Jalirgang 1926, Heft 4, p. 145.150.

(39) Vening-MeInesz F. A.: The mechanism of mountain formation in geosyncline belts. Proceed. Akad. Weetenschappen, vol. 34, 4, Amsterdam 1933.

(40) - : Gravimetric results in the Dutch East Indies in connection with mountain folding processes. Bull. Geol. Soc. American. vol. 43.

(11) -: Ergebnisse der Schwerkraftbeobachtungen auf dem Meere in den Jahren 1923-1932. Ergebnisse der kosmisclien Physik, vol. 2, N. 3, 1933.

(42) Gertu H.: Antillen, Molluken, zwei Inselbogen. Ein Vergleich des geologischen Baues und der Schwere-Anomalien. Geol. Rundschau, vol. 39, 1951, Heft 1, p. 273.284 .

(43) Kunnke PII, P. The negative isostatic anomalies in the East Indies (with experiments). Leidsche Geol. Med. dl. 8, afl. 2, 1936, p. 169-224.

(14) Grigcs D.: A theory of mountain building. American Journ. Science, September 1939, p. 611.650.

(45) Tromp S. W.: On the mechanism of the geologicnl undulation phenomenon in general and of folding in particular and their application to the problem of the roots of mountains theory. Leiden 1937, p. 1-184.

(16) Vening-Meinesz: Convection currents in the Earth. Proceed. Ned. Akad. v. Weetensch. Ib, 50, 3 .

(4i) PP. Marre \& Boscovich: Joyage astronomique et géographic dans l'État de l'Eglise. Traduit du Latin. Paris 1770. Livre cinquième Reserches sur la figure de la Terre, déterminé par les lois de l'équilibre, et par la mesure des degrés, p. $361-500$.

(18) Barrace C.: Observations on the Temple of Serapis. at Pozzuoli, near Naples, wirth remarks on certain causes which may produce Geological Cycles of great extent. Quaterly Journ. Geol. Soc. Vol. IlI, 1817, p. 186-217.

(49) READE T. M.: The origin of mountain ranges, considerd experimentally, structurally and dynamically. London 1886. 359 pages, 42 plates.

(50) Onstrand C. E.: Some possible application of geothermic to geology. Bull. American Association Peproleum Geologists, vol. 18, N. 1, January 1934, p. 13-38.

(51) JefFneys II.: The Earth, its origin, history and physical conditions. Cambridge 1929 , p. 154 .

(52) Festa C. e Santangelo M.: La radioattività della Terra. Annali di Geofisica, vol. I, 1918, p. 581.609.

(53) Von WolfF F.: Stoff und Zustand im Innern der Erde. Nova Acta Leopoldina. Neue Folge, vol. 12, N. 87, p. 383-402. 
(51) Rosmaci O.: Theoretische Untersurhungen zum Problem der Isostasie. Geofisica pura e applicata. Vol, 22, Fasc. 1-2, 1952.

(5ii) Biscinof G.: On the nutural history of vulcunos and earthquakes. Edinburgh New Plil. Journ. October 1838-January 1839, vol. 51-52, p. 25-81.

(56) DeELY R. M.: Gebirgsbildung durch Hebung tiefer Schichten mit hober Teniperatur. Geol. Magazine, vol. Vil, 1910, p. 501-503.

(5i) Hopkins W.: Researches in physical geology. Trans. Cambridge Phil. Soc., vol. VI, part I, 1836, p. 1-8t.

(58) Reiv H. F.: Isostasy and Mountain Ranges. Proceed. American Phil. Soc, vol. I, 1911 , p. $444-451$.

(5i) Dutron C.. E.: On some of the greater problems of physical geology. Rull. Phil. Soc. of. Washington. Yol. XI, p. 51-6.1. Read before the society, April 27, 1889. 